\title{
A Resistive Boundary Condition Enhanced DGTD Scheme for the Transient Analysis of Graphene
}

\author{
Ping Li, Member, IEEE, Li Jun Jiang, Senior Member, IEEE and Hakan Bağc1, Senior Member, IEEE
}

\begin{abstract}
In this paper, the electromagnetic (EM) features of graphene are characterized by a discontinuous Galerkin timedomain (DGTD) algorithm with a resistive boundary condition (RBC). The atomically thick graphene is equivalently modeled using a $\mathrm{RBC}$ by regarding the graphene as an infinitesimally thin conductive sheet. To incorporate RBC into the DGTD analysis, the surface conductivity of the graphene composed of contributions from both intraband and interband terms is firstly approximated by rational basis functions using the fastrelaxation vector-fitting (FRVF) method in the Laplace-domain. Next, through the inverse Laplace transform, the corresponding time-domain matrix equations in integral can be obtained. Finally, these matrix equations are solved by time-domain finite integral technique (FIT). For elements not touching the graphene sheet, however, the well-known Runge-Kutta (RK) method is employed to solve the two first-order time-derivative Maxwell's equations. The application of the surface boundary condition significantly alleviates the memory consuming and the limitation of time step size required by Courant-Friedrichs-Lewy (CFL) condition. To validate the proposed algorithm, various numerical examples are presented and compared with available references.
\end{abstract}

Index Terms-Graphene, surface conductivity, resistive boundary condition (RBC), discontinuous Galerkin time-domain (DGTD) method, fast-relaxation vector-fitting (FRVF), Laplace transform, finite integral technique (FIT).

\section{INTRODUCTION}

G RAPHENE, an atomically thick two-dimensional (2D) layer of carbon atoms in which the atoms are arranged in a honeycomb lattice, has already gained intensive attentions from the academia and industry communities because of its unique electrical (electron mobility up to $200,000 \mathrm{~cm}^{2} / \mathrm{V}_{\mathrm{s}}$ for suspended graphene), mechanical (Young's modulus up to $1 \mathrm{TPa}$ ), and thermal (thermal conductivity up to $5000 \mathrm{~W} / \mathrm{mK}$ ) properties [1]. These remarkable features make it as a promising candidate for semiconductors, tuneable nanoantennas, and surface plasmon waveguides, etc. The surface conductivity of graphene denoted as $\sigma_{g}\left(\omega, \mu_{c}, \Gamma, T\right)$ plays pivotal roles in studying its electromagnetic/optical properties such as surface plasmon polarization (SPP) and transformation of optics [2], where $\sigma_{g}$ is a function of temperature $T$, chemical potential

This work was supported in part by the Research Grants Council of Hong Kong (GRF 712612 and 711511), NSFC 61271158, US AR AOARD 124082 and 134140, and Hong Kong UGC AoE/PC04/08.

P. Li and L. J. Jiang are with the Department of Electrical and Electronic Engineering, The University of Hong Kong, Hong Kong, China (liping@eee.hku.hk,jianglj@hku.hk).

P. Li and H. Bağc1 are with the Division of Computer, Electrical, and Mathematical Sciences and Engineering and the Center for Uncertainty Quantification in Computational Science and Engineering, King Abdullah University of Science and Technology (KAUST), Thuwal, 23955-6900, Saudi Arabia (ping.li@kaust.edu.sa, hakan.bagci@kaust.edu.sa). or Fermi level $\mu_{c}$ (dependent on carrier density, electrostatic biasing, chemical doping), particle scattering rate $\Gamma$ [3]. In the absence of external magnetostatic biasing, $\sigma_{g}$ is a scalar, otherwise it becomes a tensor [4]-[6] due to the quantum Hall effect. By dynamically tuning the surface conductivity, the propagation, polarization, radiation, and scattering of electromagnetic waves through graphene can be manipulated conveniently.

The graphene's surface conductivity $\sigma_{g}$ consists of contributions from two terms: intraband $\sigma_{\text {intra }}$ and interband $\sigma_{\text {inter }}$. To quantify the EM properties of graphene, various numerical algorithms have been developed, including the method of moments (MoM) [7] and finite-difference timedomain (FDTD) method [8]. Compared with frequency domain methods [7], time-domain methods [3], [8], [9] have a plethora of advantages such as broadband characterization with only a single simulation and transient response capture, etc. Generally, there are two approaches to model the graphene: i) The graphene is considered as a thin layer with nanoscale finite thickness (around $0.34 \mathrm{~nm}$ ) [10], [11], thus volumetric meshes are required. With this approach, the surface conductivity is transformed to an equivalent permittivity. ii) The graphene is modeled as a infinitesimal thin conductive sheet over which a resistive boundary condition (RBC) is satisfied [8], [9]. For the first approach, particularly fine spatial mesh elements are unavoidable, which results in extremely stringent time step size. Doubtlessly, it significantly increases the memory load and CPU time. Nevertheless, by exploiting the RBC, the second approach is free of these negatives.

In this work, a discontinuous Galerkin time-domain (DGTD) [16]-[18] method is introduced to characterize the graphene based upon the RBC. DGTD method integrates the merits of both finite-volume method (FVM) [12] and finite element method (FEM) [13]. Like FVM, all operations of DGTD are local since the information exchange among neighboring elements are enforced through the numerical flux, thus resulting in block-diagonal mass matrices. The dimension of each block is equal to the degree of freedoms (DoF) in each element. Mass-matrix blocks are inverted and stored before time marching which produces a very compact and efficient solver when it is combined with an explicit scheme.

Since all boundary conditions in DGTD are implemented by redefining the Rankine-Hugoniot jump relations, thus the new numerical flux formulation in the presence of the RBC must be derived. Unfortunately, the mapping-pair of Maxwell's equations gauged by the RBC from the frequency-domain to the time-domain is not available since the surface conductivity $\sigma_{g}$ is a very complicated function of the angular frequency $\omega$ [see 
(3) and (4)]. To address this issue, the surface conductivity $\sigma_{g}$ is firstly approximated by a set of rational basis functions using a fast-relaxation vector-fitting (FRVF) [14]-[16] technique in the Laplace-domain. Via the inverse Laplace transform, the corresponding time-domain matrix equations can be obtained in integral forms over the time variable $t$ due to the fact that the division of a function by the Laplace state variable $s(s=j \omega)$ is an integral operation to the function in the time domain [16], [18], i.e.,

$$
s^{-n} F(s) \leftrightarrow \underbrace{\int \cdots \int}_{n} f(\tau) \underbrace{d \tau \cdots d \tau}_{n}
$$

These finalized Maxwell's equations in integral forms will be discretized through a finite integral technique (FIT) in the temporal sequence. It is necessary to indicate that those mesh elements not touching the graphene sheet are solved by the standard fourth-order Runge-Kutta (RK) marching scheme since the numerical flux is same as that in the absence of RBC.

The remainder of this paper is organized as follows. In Section II, the theory and formulation of the proposed algorithm are detailed, including the description of RBC, the derivation of numerical flux, and the formulation of matrix equations. In Section III, numerical results are presented for illustration. Conclusions are presented at the end of this paper.

\section{THEORY AND FORMULATION}

\section{A. Resistive Boundary Condition}

The 2D graphene layer is considered as an infinitely conductive thin sheet quantified by a scalar surface conductivity $\sigma_{g}\left(\omega, \mu_{c}, \Gamma, T\right)$ in the absence of external magnetostatic biasing field. According to the Kubo's formula [19], the surface conductivity $\sigma_{g}$ is formulated as:

$$
\begin{aligned}
\sigma_{g}\left(\omega, \mu_{c}, \Gamma, T\right) & =\frac{j q^{2}}{\pi \hbar(\omega-j 2 \Gamma)} \int_{0}^{\infty} \varepsilon\left[\frac{\partial f_{d}(\varepsilon)}{\partial \varepsilon}-\frac{\partial f_{d}(-\varepsilon)}{\partial \varepsilon}\right] d \varepsilon \\
& -\frac{j q^{2}(\omega-j 2 \Gamma)}{\pi \hbar^{2}} \int_{0}^{\infty} \frac{f_{d}(-\varepsilon)-f_{d}(\varepsilon)}{(\omega-j 2 \Gamma)^{2}-4(\varepsilon / \hbar)^{2}} d \varepsilon
\end{aligned}
$$

where $\varepsilon$ indicates the energy state, $\hbar$ denotes the reduced Planck's constant, $-q$ is the charge of electron, $f_{d}(\varepsilon)=$ $\left[e^{\left(\varepsilon-\mu_{c}\right) / k_{B} T}+1\right]^{-1}$ is the Fermi-Dirac distribution with the Boltzmann's constant $k_{B}$. In (2), the first term corresponding to the contribution from the intraband is evaluated as

$$
\sigma_{\text {intra }}=-\frac{j q^{2} k_{B} T}{\pi \hbar^{2}(\omega-j 2 \Gamma)}\left[\frac{\mu_{c}}{k_{B} T}+2 \ln \left(\mathrm{e}^{-\mu_{\mathrm{c}} / \mathrm{k}_{\mathrm{B}} \mathrm{T}}+1\right)\right]
$$

while the second term in (2) resembling the interband contribution is approximated, for $k_{B} T \ll \hbar \omega$ and $\left|\mu_{c}\right|$, by

$$
\sigma_{\text {inter }}=-\frac{j q^{2}}{4 \pi \hbar} \ln \left[\frac{2\left|\mu_{\mathrm{c}}\right|-(\omega-\mathrm{j} 2 \Gamma) \hbar}{2\left|\mu_{\mathrm{c}}\right|+(\omega-\mathrm{j} 2 \Gamma) \hbar}\right]
$$

As a resistive thin sheet with the finite conductivity $\sigma_{g}$, the electric current density is proportional to the tangential electric field at its surface. Based on this fact, the boundary condition across the graphene sheet should satisfy

$$
\begin{aligned}
& \hat{\mathbf{n}} \times\left(\mathbf{E}^{2}-\mathbf{E}^{1}\right)=0 \\
& \hat{\mathbf{n}} \times\left(\mathbf{H}^{2}-\mathbf{H}^{1}\right)=\sigma_{g} \mathbf{E}_{t}
\end{aligned}
$$

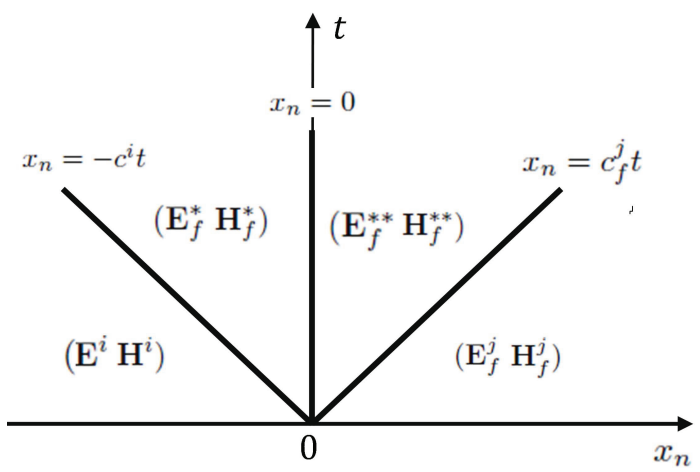

Fig. 1. Schematic illustration of the Rankine-Hugoniot jump relations for the mesh element $i$. If face $f$ of element $i$ touches the graphene, the jump relation over this face along the curve $x_{n}=0$ should meet the relation in (10). Otherwise, both electric and magnetic fields are tangentially continuous. The horizontal axis represents the normal direction over the element's face, the vertical axis $t$ denotes the time [12].

where the superscripts 1 and 2 represent the two sides of the graphene sheet, $\hat{\mathbf{n}}$ is a unit vector pointing normal to the graphene sheet, $\mathbf{E}_{t}=\hat{\mathbf{n}} \times(\mathbf{E} \times \hat{\mathbf{n}})$ is the tangential component of $\mathbf{E}$ field along the graphene sheet where $\mathbf{E}$ is approximated by $\mathbf{E}=\left(\mathbf{E}^{1}+\mathbf{E}^{2}\right) / 2$.

It is necessary to emphasize that the boundary condition in (5) and (6) are derived based on the fact that the skin-depth is much larger than the thickness of the conductive sheet. It is independent of the external excitations such as the incident directions and polarizations of plane waves, etc.

\section{B. Formulation of the Upwind Flux}

In DGTD, the enforcement of boundary conditions in (5) and (6) is facilitated via reformulating the numerical flux based on the Rankine-Hugoniot jump relations. For an arbitrary mesh element $i$ (tetrahedrons are used as the mesh elements in this work), the jump relations along three characteristic [12] for the $f$-th face of element $i$ shown in Fig. 1 are defined as (assume the face $f_{g}$ of element $i$ is over the graphene sheet):

1) Jump across the characteristic $x_{n}=-c^{i} t$

$$
\begin{gathered}
\frac{1}{\mu^{i}} \hat{\mathbf{n}}_{i, f} \times\left(\mathbf{E}_{f}^{*}-\mathbf{E}^{i}\right)=-c^{i}\left(\mathbf{H}_{f}^{*}-\mathbf{H}^{i}\right) \\
\frac{1}{\epsilon^{i}} \hat{\mathbf{n}}_{i, f} \times\left(\mathbf{H}^{i}-\mathbf{H}_{f}^{*}\right)=-c^{i}\left(\mathbf{E}_{f}^{*}-\mathbf{E}^{i}\right)
\end{gathered}
$$

2) Jump across the characteristic $x_{n}=0$

$$
\begin{aligned}
& \hat{\mathbf{n}}_{i, f} \times\left(\mathbf{E}_{f}^{* *}-\mathbf{E}_{f}^{*}\right)=0 \\
& \hat{\mathbf{n}}_{i, f} \times\left(\mathbf{H}_{f}^{* *}-\mathbf{H}_{f}^{*}\right)=\alpha_{g} \sigma_{g} \mathbf{E}_{t}
\end{aligned}
$$

3) Jump across the characteristic $x_{n}=c_{f}^{j} t$

$$
\begin{gathered}
\frac{1}{\mu_{f}^{j}} \hat{\mathbf{n}}_{i, f} \times\left(\mathbf{E}_{f}^{j}-\mathbf{E}_{f}^{* *}\right)=c_{f}^{j}\left(\mathbf{H}_{f}^{j}-\mathbf{H}_{f}^{* *}\right) \\
\frac{1}{\epsilon_{f}^{j}} \hat{\mathbf{n}}_{i, f} \times\left(\mathbf{H}_{f}^{* *}-\mathbf{H}_{f}^{j}\right)=c_{f}^{j}\left(\mathbf{E}_{f}^{j}-\mathbf{E}_{f}^{* *}\right)
\end{gathered}
$$

where $1 \leq f \leq 4$ for tetrahedrons, $j$ denotes the neighboring element sharing the $f$-th face of element $i, \hat{\mathbf{n}}_{i, f}$ is the outward 
unit normal vector of face $f . c^{i}$ and $c_{f}^{j}$ represent the characteristic speed in element $i$ and its neighboring $j$, respectively. $\epsilon^{i / j}$ and $\mu^{i / j}$ are the permittivity and permeability, respectively. $\left(\mathbf{E}_{f}^{*}, \mathbf{H}_{f}^{*}\right)$ and $\left(\mathbf{E}_{f}^{* *}, \mathbf{H}_{f}^{* *}\right)$ are the intermediate states in element $i$ and $j$, respectively. It is noted that the incorporation of RBC is facilitated by disrupting the tangential continuity of intermediate variables $\mathbf{H}_{f}^{*}$ and $\mathbf{H}_{f}^{* *}$. The above jump relations have to be satisfied over the faces shared by element $i$ and $j$. The parameter $\alpha_{g}$ is defined as

$$
\alpha_{g}=\left\{\begin{array}{l}
1, f=f_{g} \\
0, f \neq f_{g}
\end{array}\right.
$$

By combining (7), (9), (10), and (11), the upwind flux for the Ampere's law equation is formulated by

$$
\begin{aligned}
\hat{\mathbf{n}}_{i, f} \times \mathbf{H}_{f}^{*}= & \hat{\mathbf{n}}_{i, f} \times\left[\frac{\left(Z^{i} \mathbf{H}^{i}+Z_{f}^{j} \mathbf{H}_{f}^{j}\right)+\hat{\mathbf{n}}_{i, f} \times\left(\mathbf{E}^{i}-\mathbf{E}_{f}^{j}\right)}{Z^{i}+Z_{f}^{j}}\right. \\
& \left.+\alpha_{g} \frac{Z^{j} \sigma_{g} \hat{\mathbf{n}}_{i, f} \times\left(\mathbf{E}^{i}+\mathbf{E}_{f}^{j}\right)}{2\left(Z^{i}+Z_{f}^{j}\right)}\right]
\end{aligned}
$$

Similarly, with (8), (9), (10) and (12), the upwind flux for the Maxwell-Faraday's law equation is given by

$$
\begin{aligned}
\hat{\mathbf{n}}_{i, f} \times \mathbf{E}_{f}^{*}= & \hat{\mathbf{n}}_{i, f} \times\left[\frac{\left(Y^{i} \mathbf{E}^{i}+Y_{f}^{j} \mathbf{E}_{f}^{j}\right)+\hat{\mathbf{n}}_{i, f} \times\left(\mathbf{H}_{f}^{j}-\mathbf{H}^{i}\right)}{Y^{i}+Y_{f}^{j}}\right. \\
& \left.-\alpha_{g} \frac{\sigma_{g}\left(\mathbf{E}^{i}+\mathbf{E}_{f}^{j}\right)}{2\left(Y^{i}+Y_{f}^{j}\right)}\right]
\end{aligned}
$$

where $Z^{i}=\sqrt{\mu^{i} / \epsilon^{i}}$ and $Z_{f}^{j}=\sqrt{\mu_{f}^{j} / \epsilon_{f}^{j}}$ represent the characteristic impedance of element $i$ and the neighboring at the $f$-th face. By substituting (3) and (4) into (14) and (15), obviously, straightforward mapping the DG formulated Maxwell's equations from the frequency-domain to time-domain is not possible due to the lack of analytical Fourier transform pair. To overcome this problem, we firstly approximate the surface conductivity $\sigma_{g}$ by rational functions in the Laplace-domain, then via inverse Laplace transform, time-domain counterparts can be obtained conveniently.

\section{Fast-Relaxation Vector-Fitting Technique}

Suppose a given group of samples $\left\{\left(\omega_{p}, \sigma_{g}\left(\omega_{p}\right), p=\right.\right.$ $1, \cdots, P\}$ represent the values of surface conductivity $\sigma_{g}$ at angular frequency $\omega_{p}$, FRVF [14], [15] technique is employed to fit these samples by rational basis functions. Namely,

$$
\begin{aligned}
\sigma_{g}(s) & =\sum_{m}^{M} \frac{c_{m}}{s-a_{m}}+d+s e \\
& =\sum_{m}^{M} \frac{c_{m} s^{-1}}{1-a_{m} s^{-1}}+d+\frac{e}{s^{-1}}
\end{aligned}
$$

where $s$ is the Laplace state variable, $a_{m}$ and $c_{m}$ (which can be either real quantities or complex pairs) denote the pole and residue, respectively. $d$ and $e$ are two optional real parameters, which are set to zero in this work. Then, we can rewrite (16) as:

$$
\begin{aligned}
\sigma_{g}(s) & =\frac{u_{1} s^{-1}+u_{2} s^{-2}+\cdots+u_{P} s^{-P}}{d_{0}+d_{1} s^{-1}+d_{2} s^{-2}+\cdots+d_{Q} s^{-Q}} \\
& =\frac{\sum_{p=1}^{P} u_{p} s^{-p}}{\sum_{q=0}^{Q} d_{q} s^{-q}}
\end{aligned}
$$

where $u_{p}$ and $d_{q}$ are the $p$ - and $q$-th coefficients, and the orders of numerator and denominator are $P$ and $Q$, respectively.

To fix the poles and residues, FRVF involves two stages: pole identification and residue identification. With this rational function and the transform pair in (1), the corresponding timedomain equations are obtained in an integral form to time $t$. The integral operators will be further discretized by FIT in this work.

\section{Formulation of DGTD}

Let $\Omega$ denote the computation domain of interest, which is bound by the surface $\partial \Omega$. For DGTD, the domain $\Omega$ is firstly split into $N$ non-overlapping tetrahedrons $\Omega_{i}$ with the corresponding boundary $\partial \Omega_{i}$. In element $i$, the electric field $\mathbf{E}$ and magnetic field $\mathbf{H}$ are expanded by vector basis functions $\boldsymbol{\Phi}(\mathbf{r})$ and $\boldsymbol{\Psi}(\mathbf{r})$ [16], [20], [27]:

$$
\begin{aligned}
\mathbf{E}^{i} & =\sum_{k=1}^{n_{e}^{i}} e_{k}^{i}(t) \boldsymbol{\Phi}_{k}^{i}(\mathbf{r}) \\
\mathbf{H}^{i} & =\sum_{l=1}^{n_{h}^{i}} h_{l}^{i}(t) \Psi_{l}^{i}(\mathbf{r})
\end{aligned}
$$

where $n_{e}^{i}$ and $n_{h}^{i}$ are the number of vector basis functions for $\mathbf{E}$ and $\mathbf{H}$ in the $i$-th element, $e_{k}^{i}$ and $h_{k}^{i}$ are the unknown time-dependent coefficients of basis functions.

Next, the mesh cells are regrouped into two different sets $\Omega_{\mathrm{RBC}}$ and $\Omega_{\mathrm{NRBC}}$. The set $\Omega_{\mathrm{RBC}}$ consists of mesh elements interfacing with the graphene sheet (or RBC), while the set $\Omega_{\mathrm{NRBC}}$ contains those meshes not touching the graphene sheet (or RBC). For elements in $\Omega_{\mathrm{NRBC}}$, the fourth-order RK method is employed to solve the two first-order time derivative Maxwell's equations [17]; while for elements in $\Omega_{\mathrm{RBC}}$, FIT is applied to discrete the integral operators. Since RK marching scheme based DGTD formulation is already well developed [16], [17], [24], we only detail the FIT based DGTD formulation in this work. To obtain the time-domain matrix equations, we start with the two first-order Maxwell's equations in the Laplace-domain.

Suppose that the element $i$ belongs to the set $\Omega_{\mathrm{RBC}}$, and its $f$-th $(f \in \mathbb{N}, 1 \leq f \leq 4$ for tetrahedron) face overlaps with the graphene sheet. By applying the DG testing over the two 
Maxwell's curl equations in Laplace domain, we can obtain

$$
\begin{aligned}
& \int_{\Omega_{i}} \boldsymbol{\Phi}_{k}^{i} \cdot\left[\epsilon_{i} \mathbf{E}^{i}-s^{-1} \nabla \times \mathbf{H}^{i}\right] d \mathbf{r}= \\
& s^{-1} \sum_{f=1}^{4} \int_{\partial \Omega_{i, f}} \boldsymbol{\Phi}_{k}^{i} \cdot\left[\hat{\mathbf{n}}_{i, f} \times\left(\mathbf{H}_{f}^{*}-\mathbf{H}^{i}\right)\right] d \mathbf{r} \\
& \int_{\Omega_{i}} \boldsymbol{\Psi}_{l}^{i} \cdot\left[\mu_{i} \mathbf{H}^{i}+s^{-1} \nabla \times \mathbf{E}_{i}\right] d \mathbf{r}= \\
& s^{-1} \sum_{f=1}^{4} \int_{\partial \Omega_{i, f}} \mathbf{\Psi}_{l}^{i} \cdot\left[\hat{\mathbf{n}}_{i, f} \times\left(\mathbf{E}^{i}-\mathbf{E}_{f}^{*}\right)\right] d \mathbf{r}
\end{aligned}
$$

With (14)-(21) and the inverse Laplace transform in (1), the time-domain matrix equations can be derived as [?]:

$$
\begin{aligned}
& \mathbf{M}_{\mathrm{e}}^{i}\left(d_{0} \mathbf{e}_{0}^{i}+d_{1} \mathbf{e}_{1}^{i}+\cdots+d_{Q} \mathbf{e}_{Q}^{i}\right) \\
& -\mathbf{S}_{\mathrm{e}}^{i}\left(d_{0} \mathbf{h}_{1}^{i}+d_{1} \mathbf{h}_{2}^{i}+\cdots+d_{Q} \mathbf{h}_{Q+1}^{i}\right) \\
& =\sum_{f=1}^{4}\left(\mathbf{F}_{e e}^{i i, f} \mathbf{e}_{f}^{i}+\mathbf{F}_{e e}^{i j, f} \mathbf{e}_{f}^{j}+\mathbf{F}_{e h}^{i i, f} \mathbf{h}_{f}^{i}+\mathbf{F}_{e h}^{i j, f} \mathbf{h}_{f}^{j}\right) \\
& +\underbrace{\mathbf{F}_{e e}^{i j, \sigma_{g}} \mathbf{e}^{j, \sigma_{g}}+\mathbf{F}_{e e}^{i i, \sigma_{g}} \mathbf{e}^{i, \sigma_{g}}}_{\mathbf{F}_{\mathrm{e}}^{\sigma_{g}}} \\
& \mathbf{M}_{\mathrm{h}}^{i}\left(d_{0} \mathbf{h}_{0}^{i}+d_{1} \mathbf{h}_{1}^{i}+\cdots+d_{Q} \mathbf{h}_{Q}^{i}\right) \\
& +\mathbf{S}_{\mathrm{h}}^{i}\left(d_{0} \mathbf{e}_{1}^{i}+d_{1} \mathbf{e}_{2}^{i}+\cdots+d_{Q} \mathbf{e}_{Q+1}^{i}\right) \\
& =\sum_{f=1}^{4}\left(\mathbf{F}_{h h}^{i i, f} \mathbf{h}_{f}^{i}+\mathbf{F}_{h h}^{i j, f} \mathbf{h}_{f}^{j}+\mathbf{F}_{h e}^{i i, f} \mathbf{e}_{f}^{i}+\mathbf{F}_{h e}^{i j, f} \mathbf{e}_{f}^{j}\right) \\
& +\underbrace{\mathbf{F}_{h e}^{i j, \sigma_{g}} \mathbf{e}^{j, \sigma_{g}}+\mathbf{F}_{h e}^{i i, \sigma_{g}} \mathbf{e}^{i, \sigma_{g}}}_{\mathbf{F}_{\mathrm{h}}^{\sigma_{g}}}
\end{aligned}
$$

where $\mathbf{M}_{\mathrm{e} / \mathrm{h}}^{i}$ and $\mathbf{S}_{e / h}^{i}$ are mass and stiffness matrices with dimension equal to the DoF in the element $i, \mathbf{F}_{e e}, \mathbf{F}_{e h}, \mathbf{F}_{h h}$, and $\mathbf{F}_{h e}$ are matrices arising from the numerical flux terms. They are defined as follows:

$$
\begin{gathered}
{\left[\mathbf{M}_{e}^{i}\right]_{k l}=\int_{\Omega_{i}} \boldsymbol{\Phi}_{k}^{i} \cdot \epsilon^{i} \mathbf{\Phi}_{l}^{i} d \mathbf{r}} \\
{\left[\mathbf{M}_{h}^{i}\right]_{k l}=\int_{\Omega_{i}} \mathbf{\Psi}_{k}^{i} \cdot \mu^{i} \mathbf{\Psi}_{l}^{i} d \mathbf{r}} \\
{\left[\mathbf{S}_{e}^{i}\right]_{k l}=\int_{\Omega_{i}} \boldsymbol{\Phi}_{k}^{i} \cdot \nabla \times \mathbf{\Psi}_{l}^{i} d \mathbf{r}} \\
{\left[\mathbf{F}_{e e}^{i i, f}\right]_{k l}=\frac{-1}{Z^{i}+Z_{f l}^{j}}=\int_{\partial \Omega_{i, f}} \boldsymbol{\Phi}_{k}^{i} \cdot \hat{\mathbf{n}}_{i, f}^{i} \times\left(\hat{\mathbf{n}}_{i, f} \times \mathbf{\Phi}_{l}^{i}\right) d \mathbf{r}} \\
{\left[\mathbf{F}_{e e}^{i j, f}\right]_{k l}=\frac{1}{Z^{i}+Z_{f}^{j}} \int_{\partial \Omega_{i, f}} \boldsymbol{\Phi}_{k}^{i} \cdot \hat{\mathbf{n}}_{i, f} \times\left(\hat{\mathbf{n}}_{i, f} \times \mathbf{\Phi}_{l}^{j, f}\right) d \mathbf{r}} \\
{\left[\mathbf{F}_{e h}^{i i, f}\right]_{k l}=-\frac{Z_{f}^{j}}{Z^{i}+Z_{f}^{j}} \int_{\partial \Omega_{i, f}} \boldsymbol{\Phi}_{k}^{i} \cdot \hat{\mathbf{n}}_{i, f} \times \mathbf{\Psi}_{l}^{i} d \mathbf{r}}
\end{gathered}
$$

$$
\begin{gathered}
{\left[\mathbf{F}_{e h}^{i j, f}\right]_{k l}=\frac{Z_{f}^{j}}{Z^{i}+Z_{f}^{j}} \int_{\partial \Omega_{i, f}} \boldsymbol{\Phi}_{k}^{i} \cdot \hat{\mathbf{n}}_{i, f} \times \mathbf{\Psi}_{l}^{j, f} d \mathbf{r}} \\
{\left[\mathbf{F}_{e e}^{i i, \sigma_{g}}\right]_{k l}=\frac{Z_{f_{g}}^{j}}{2\left(Z^{i}+Z_{f_{g}}^{j}\right)} \int_{\partial \Omega_{i, f_{g}}} \boldsymbol{\Phi}_{k}^{i} \cdot \hat{\mathbf{n}}_{i, f_{g}} \times\left(\hat{\mathbf{n}}_{i, f_{g}} \times \mathbf{\Phi}_{l}^{i}\right) d \mathbf{r}} \\
{\left[\mathbf{F}_{e e}^{i j, \sigma_{g}}\right]_{k l}=\frac{Z_{f_{g}}^{j}}{2\left(Z^{i}+Z_{f_{g}}^{j}\right)} \int_{\partial \Omega_{i, f_{g}}} \boldsymbol{\Phi}_{k}^{i} \cdot \hat{\mathbf{n}}_{i, f_{g}} \times\left(\hat{\mathbf{n}}_{i, f_{g}} \times \mathbf{\Phi}_{l}^{j, f_{g}}\right) d \mathbf{r}} \\
{\left[\mathbf{F}_{h h}^{i i, f}\right]_{k l}=\frac{-1}{Y^{i}+Y_{f}^{j}} \int_{\partial \Omega_{i, f}} \mathbf{\Psi}_{k}^{i} \cdot \hat{\mathbf{n}}_{i, f} \times\left(\hat{\mathbf{n}}_{i, f} \times \mathbf{\Psi}_{l}^{i}\right) d \mathbf{r}} \\
{\left[\mathbf{F}_{h h}^{i j, f}\right]_{k l}=\frac{1}{Y^{i}+Y_{f}^{j}} \int_{\partial \Omega_{i, f}} \mathbf{\Psi}_{k}^{i} \cdot \hat{\mathbf{n}}_{i, f} \times\left(\hat{\mathbf{n}}_{i, f} \times \mathbf{\Psi}_{l}^{j, f}\right) d \mathbf{r}} \\
{\left[\mathbf{F}_{h e}^{i i, f}\right]_{k l}=\frac{Y_{f}^{j}}{Y^{i}+Y_{f}^{j}} \int_{\partial \Omega_{i, f}} \mathbf{\Psi}_{k}^{i} \cdot \hat{\mathbf{n}}_{i, f} \times \mathbf{\Phi}_{l}^{i} d \mathbf{r}} \\
{\left[\mathbf{F}_{h e}^{i j, f}\right]_{k l}=\frac{-Y_{f}^{j}}{Y^{i}+Y_{f}^{j}} \int_{\partial \Omega_{i, f}} \mathbf{\Psi}_{k}^{i} \cdot \hat{\mathbf{n}}_{i, f} \times \mathbf{\Phi}_{l}^{j, f} d \mathbf{r}} \\
{\left[\mathbf{F}_{h e}^{i i, \sigma_{g}}\right]_{k l}=\frac{1}{2\left(Y^{i}+Y_{f_{g}}^{j}\right)} \int_{\partial \Omega_{i, f_{g}}} \mathbf{\Psi}_{k}^{i} \cdot\left(\hat{\mathbf{n}}_{i, f_{g}} \times \mathbf{\Phi}_{l}^{i}\right) d \mathbf{r}} \\
{\left[\mathbf{F}_{h e}^{i j, \sigma_{g}}\right]_{k l}=\frac{1}{2\left(Y^{i}+Y_{f_{g}}^{j}\right)} \int_{\partial \Omega_{i, f_{g}}} \mathbf{\Psi}_{k}^{i} \cdot\left(\hat{\mathbf{n}}_{i, f_{g}} \times \mathbf{\Phi}_{l}^{j, f_{g}}\right) d \mathbf{r}}
\end{gathered}
$$

It should be noted that the flux terms $\mathbf{F}_{e}^{\sigma_{g}}=\mathbf{F}_{e e}^{i j, \sigma_{g}}+\mathbf{F}_{e e}^{i i, \sigma_{g}}$ and $\mathbf{F}_{h}^{\sigma_{g}}=\mathbf{F}_{h e}^{i j, \sigma_{g}}+\mathbf{F}_{h e}^{i i, \sigma_{g}}$ are not zero only for faces over the graphene sheet. Otherwise, they are zero.

Moreover, terms $\mathbf{e}_{q}^{i}$ and $\mathbf{h}_{q}^{i}$ are column vectors storing the unknown coefficients $\mathrm{e}_{k}^{i}$ and $\mathrm{h}_{l}^{i}$ and defined as

$$
\begin{aligned}
\mathbf{e}_{q, k}^{i} & =\underbrace{\int \cdots \int}_{q} \mathrm{e}_{k}^{i}(\tau) \underbrace{d \tau \cdots d \tau}_{q} \\
\mathbf{h}_{q, l}^{i} & =\underbrace{\int \cdots \int}_{q} \mathrm{~h}_{l}^{i}(\tau) \underbrace{d \tau \cdots d \tau}_{q}
\end{aligned}
$$

The expressions for other parameters in (22) and (23) are

$$
\begin{aligned}
& (\mathbf{e} / \mathbf{h})_{f}^{i}=\sum_{q=0}^{Q} d_{q}\left(\mathbf{e}_{f}^{i} / \mathbf{h}_{f}^{i}\right)_{q+1} \\
& (\mathbf{e} / \mathbf{h})_{f}^{j}=\sum_{q=0}^{Q} d_{q}\left(\mathbf{e}_{f}^{j} / \mathbf{h}_{f}^{j}\right)_{q+1} \\
& \mathbf{e}^{i, \sigma_{g}}=\sum_{p=1}^{P} u_{p} \mathbf{e}_{p+1}^{i} \\
& \mathbf{e}^{j, \sigma_{g}}=\sum_{p=1}^{P} u_{p} \mathbf{e}_{f_{g}, p+1}^{j}
\end{aligned}
$$

Referring (40) and (41), it is noted that all terms in (42) are in the form of integration over time $t$.

To solve the matrix equations in (22) and (23), the integrations over time are discretized by FIT based upon the 
rectangular rule [?], [16]. The discretized version of (40) and (41) with order $p$ or $q$ at $t=(n+1) \delta t$ can be written as:

$$
\begin{gathered}
\mathbf{e}_{n+1, p / q}^{i}=(\delta t)^{p / q} \sum_{k_{p / q}=0}^{n} \cdots \sum_{k_{2}=0}^{k_{3}} \sum_{k_{1}=0}^{k_{2}} \mathbf{e}_{k_{1}+1}^{i} \\
\mathbf{h}_{n+1, p / q}^{i}=(\delta t)^{p / q} \sum_{k_{p / q}=0}^{n} \cdots \sum_{k_{2}=0}^{k_{3}} \sum_{k_{1}=0}^{k_{2}} \mathbf{h}_{k_{1}+1}^{i} \\
\mathbf{e}_{n+1,0}^{i}=\mathbf{e}_{n+1}^{i} \\
\mathbf{h}_{n+1,0}^{i}=\mathbf{h}_{n+1}^{i}
\end{gathered}
$$

To keep an explicit time marching scheme, the terms involving contributions from neighboring elements will be discretized with a forward rectangular rule [16]. That is,

$$
\begin{aligned}
\mathbf{e}_{n+1, p / q}^{j} & =(\delta t)^{p / q} \sum_{k_{p / q=0}}^{n} \cdots \sum_{k_{2}=0}^{k_{3}} \sum_{k_{1}=0}^{k_{2}} \mathbf{e}_{k_{1}}^{j} \\
\mathbf{h}_{n+1, p / q}^{j} & =(\delta t)^{p / q} \sum_{k_{p / q}=0}^{n} \cdots \sum_{k_{2}=0}^{k_{3}} \sum_{k_{1}=0}^{k_{2}} \mathbf{h}_{k_{1}}^{j}
\end{aligned}
$$

After a lengthy mathematical manipulation, (22) and (23) are finalized into a compact matrix system at $t=(n+1) \delta t$

$$
\left(\begin{array}{cc}
\widetilde{\mathbf{M}}_{e}^{i} & \widetilde{\mathbf{S}}_{e}^{i} \\
\widetilde{\mathbf{S}}_{h}^{i} & \widetilde{\mathbf{M}}_{h}^{i}
\end{array}\right)\left[\begin{array}{c}
\mathbf{e}_{n+1}^{i} \\
\mathbf{h}_{n+1}^{i}
\end{array}\right]=\left(\begin{array}{c}
\widetilde{\mathbf{F}}_{e}^{i} \\
\widetilde{\mathbf{F}}_{h}^{i}
\end{array}\right)
$$

where the matrix-blocks are defined by

$$
\begin{aligned}
& \widetilde{\mathbf{M}}_{e}^{i}=\sum_{q=0}^{Q} d_{q}(\delta t)^{q} \mathbf{M}_{e}^{i} \sum_{q=0}^{Q} d_{q}(\delta t)^{q+1} \mathbf{F}_{e e}^{i i} \sum_{p=1}^{P} u_{p}(\delta t)^{p+1} \mathbf{F}_{e e}^{i i, \sigma_{g}} \\
& \widetilde{\mathbf{S}}_{e}^{i}=-\sum_{q=0}^{Q} d_{q}(\delta t)^{q+1} \mathbf{S}_{e}^{i}+\sum_{q=0}^{Q} d_{q}(\delta t)^{q+1} \mathbf{F}_{e h}^{i i} \\
& \widetilde{\mathbf{M}}_{h}^{i}=\sum_{q=0}^{Q} d_{q}(\delta t)^{q} \mathbf{M}_{h}^{i}-\sum_{q=0}^{Q} d_{q}(\delta t)^{q+1} \mathbf{F}_{h h}^{i i} \\
& \widetilde{\mathbf{S}}_{h}^{i}=\sum_{q=0}^{Q} d_{q}(\delta t)^{q} \mathbf{S}_{h}^{i}-\sum_{q=0}^{Q} d_{q}(\delta t)^{q+1} \mathbf{F}_{h e}^{i i}-\sum_{p=1}^{P} u_{p}(\delta t)^{p+1} \mathbf{F}_{h e}^{i i, \sigma_{g}}
\end{aligned}
$$

and the column vectors are formulated as

$$
\begin{aligned}
& \widetilde{\mathbf{F}}_{e}^{i}=-\mathbf{M}_{e}^{i}\left\{\sum_{q=0}^{Q} d_{q}(\delta t)^{q} \widetilde{\mathbf{e}}_{n+1, q}^{i}\right\}+\mathbf{S}_{e}^{i}\left\{\sum_{q=0}^{Q} d_{q}(\delta t)^{q+1} \widetilde{\mathbf{h}}_{n+1, q+1}^{i}\right\} \\
& +\mathbf{F}_{e e}^{i i}\left\{\sum_{q=0}^{Q} d_{q}(\delta t)^{q+1} \widetilde{\mathbf{e}}_{n+1, q+1}^{i}\right\}+\mathbf{F}_{e e}^{i j}\left\{\sum_{q=0}^{Q} d_{q}(\delta t)^{q+1} \mathbf{e}_{n+1, q+1}^{j}\right\} \\
& +\mathbf{F}_{e h}^{i i}\left\{\sum_{q=0}^{Q} d_{q}(\delta t)^{q+1} \widetilde{\mathbf{h}}_{n+1, q+1}^{i}\right\}+\mathbf{F}_{e h}^{i j}\left\{\sum_{q=0}^{Q} d_{q}(\delta t)^{q+1} \mathbf{h}_{n+1, q+1}^{j}\right\} \\
& +\mathbf{F}_{e e}^{i i, \sigma_{g}}\left\{\sum_{p=1}^{P} u_{p}(\delta t)^{p+1} \widetilde{\mathbf{e}}_{n+1, p+1}^{i}\right\}+\mathbf{F}_{e e}^{i j, \sigma_{g}}\left\{\sum_{p=1}^{P} u_{p}(\delta t)^{p+1} \mathbf{e}_{n+1, p+1}^{j}\right\}
\end{aligned}
$$

$$
\begin{aligned}
& \widetilde{\mathbf{F}}_{h}^{i}=-\mathbf{M}{ }_{h}^{i}\left\{\sum_{q=0}^{Q} d_{q}(\delta t)^{q} \widetilde{\mathbf{h}}_{n+1, q}^{i}\right\}-\mathbf{S}_{h}^{i}\left\{\sum_{q=0}^{Q} d_{q}(\delta t)^{q+1} \widetilde{\mathbf{e}}_{n+1, q+1}^{i}\right\} \\
& +\mathbf{F}_{h h}^{i i}\left\{\sum_{q=0}^{Q} d_{q}(\delta t)^{q+1} \widetilde{\mathbf{h}}_{n+1, q+1}^{i}\right\}+\mathbf{F}_{h h}^{i j}\left\{\sum_{q=0}^{Q} d_{q}(\delta t)^{q+1} \mathbf{h}_{n+1, q+1}^{j}\right\} \\
& +\mathbf{F}_{h e}^{i i}\left\{\sum_{q=0}^{Q} d_{q}(\delta t)^{q+1} \widetilde{\mathbf{e}}_{n+1, q+1}^{i}\right\}+\mathbf{F}_{h e}^{i j}\left\{\sum_{q=0}^{Q} d_{q}(\delta t)^{q+1} \mathbf{e}_{n+1, q+1}^{j}\right\} \\
& +\mathbf{F}_{h e}^{i i, \sigma_{g}}\left\{\sum_{p=1}^{P} u_{p}(\delta t)^{p+1} \widetilde{\mathbf{e}}_{n+1, p+1}^{i}\right\}+\mathbf{F}_{h e}^{i j, \sigma_{g}}\left\{\sum_{p=1}^{P} u_{p}(\delta t)^{p+1} \mathbf{e}_{n+1, p+1}^{j}\right\}
\end{aligned}
$$

In (54),

$$
\begin{aligned}
& \widetilde{\mathbf{e}}_{n+1, p / q}^{i}=\mathbf{e}_{n+1, p / q}^{i}-(\delta t)^{p / q} \mathbf{e}_{n+1}^{i} \\
& \widetilde{\mathbf{h}}_{n+1, p / q}^{i}=\mathbf{h}_{n+1, p / q}^{i}-(\delta t)^{p / q} \mathbf{h}_{n+1}^{i} \\
& \widetilde{\mathbf{e}}_{n+1,0}^{i}=0 \\
& \widetilde{\mathbf{h}}_{n+1,0}^{i}=0
\end{aligned}
$$

To efficiently evaluate $(\mathbf{e} / \mathbf{h})_{n+1, p / q}^{i}$ in (43) and (44), $(\mathbf{e} / \mathbf{h})_{n+1, p / q}^{j}$ in (47) and (48), and $(\widetilde{\mathbf{e}} / \widetilde{\mathbf{h}})_{n+1, p / q}^{i}$ in (55) and (56), the following recursive scheme is introduced [16]:

$$
\begin{aligned}
(\mathbf{e} / \mathbf{h})_{n+1, p / q}^{i / j} & =(\mathbf{e} / \mathbf{h})_{n, p / q}^{i / j}+\delta t \cdot(\mathbf{e} / \mathbf{h})_{n+1, p-1 / q-1}^{i / j} \\
(\widetilde{\mathbf{e}} / \widetilde{\mathbf{h}})_{n+1, p / q}^{i} & =(\mathbf{e} / \mathbf{h})_{n, p / q}^{i}+\delta t \cdot(\widetilde{\mathbf{e}} / \widetilde{\mathbf{h}})_{n+1, p-1 / q-1}^{i}
\end{aligned}
$$

The dimension of the square matrix system in (49) is $\left(n_{e}^{i}+n_{h}^{i}\right)$ by $\left(n_{e}^{i}+n_{h}^{i}\right)$. In this work, 12 edge basis functions (six constant tangential/linear normal $(\mathrm{CT} / \mathrm{LN})$ and six linear tangential/linear normal (LT/LN) basis functions, respectively) are used for both $\mathbf{E}$ and $\mathbf{H}$ in each mesh element, i.e., $n_{e}^{i}=12$ and $n_{h}^{i}=12$ for $i=1, \cdots, N$.

In DGTD analysis, the inversions of matrices are calculated and stored before time marching, thus only forward matrixvector products are involved during the time marching process. For mesh elements in $\Omega_{\mathrm{RBC}}$, the computational cost is on the order of $\left(n_{e}^{i}+n_{h}^{i}\right) \times\left(n_{e}^{i}+n_{h}^{i}\right) \times O\left(N_{\mathrm{RBC}}\right)$ with $N_{\mathrm{RBC}}$ denoting the number of elements in $\Omega_{\mathrm{RBC}}$. On the other hand, for elements in $\Omega_{\mathrm{NRBC}}$, the Maxwell-Faraday's and Amperes law equations are solved separated by the RK method, and the corresponding computational costs are on the order of $\left(n_{e}^{i} \times n_{e}^{i}\right) \times O\left(N_{\mathrm{NRBC}}\right)$ and $\left(n_{h}^{i} \times n_{h}^{i}\right) \times O\left(N_{\mathrm{NRBC}}\right)$ with $N_{\text {NRBC }}$ denoting the number of elements in $\Omega_{\text {NRBC }}$, respectively. Thus, the total computational cost is on the order of $\left(n_{e}^{i}+n_{h}^{i}\right) \times\left(n_{e}^{i}+n_{h}^{i}\right) \times O\left(N_{R B C}\right)+\left(n_{e}^{i} \times n_{e}^{i}+n_{h}^{i} \times n_{h}^{i}\right) \times$ $O\left(N_{\text {NRBC }}\right)$.

Since the resultant time-marching scheme is explicit, to ensure stability, the CFL-like condition restricting the time step size $\delta t_{D G}$ of DGTD must be satisfied. Namely,

$$
c_{0} \delta t_{D G} \leq \min \left\{l_{\min } \sqrt{\epsilon_{r} \mu_{r}} / 4(p+1)^{2}\right\}
$$

where $c_{0}$ is the free-space light speed, $p$ is the order of basis function. The details can be found in [21]-[24].

\section{Numerical Results}

In this section, various numerical examples are introduced to validate the proposed algorithm by studying the EM wave 
TABLE I

The Poles $a_{m}$ AND Residues $c_{m}$ FOR THE GRAPHENE SHEET With $\mu_{c}=0.3 \mathrm{eV}$ AND $\Gamma=0.41 \mathrm{meV} / \hbar$.

\begin{tabular}{ccc}
\hline \hline $\mathrm{m}$ & $a_{m}$ & $c_{m}$ \\
\hline 1 & $-4.0 \times 10^{8}$ & -0.2967 \\
2 & $-1.9827 \times 10^{11}$ & $3.5314 \times 10^{10}$ \\
3 & $-(0.0834-j 1.5753) \times 10^{15}$ & $(5.2290+j 0.8353) \times 10^{10}$ \\
4 & $-(0.0834+j 1.5753) \times 10^{15}$ & $(5.2290-j 0.8353) \times 10^{10}$ \\
\hline
\end{tabular}

TABLE II

The Poles $a_{m}$ AND Residues $c_{m}$ FOR THE GRAPHENE SHEET WITH $\mu_{c}=0.12 \mathrm{eV}$ AND $\Gamma=3.29 \mathrm{meV} / \hbar$.

\begin{tabular}{ccc}
\hline \hline $\mathrm{m}$ & $a_{m}$ & $c_{m}$ \\
\hline 1 & $-4.0 \times 10^{13}$ & $1.4187 \times 10^{13}$ \\
2 & $-4.8203 \times 10^{14}$ & $-1.3543 \times 10^{13}$ \\
3 & $-1.3231 \times 10^{17}$ & $8.2294 \times 10^{15}$ \\
4,5 & $-(0.6734 \mp j 3.6348) \times 10^{14}$ & $-(0.0771 \mp j 1.2713) \times 10^{12}$ \\
6,7 & $-(2.0046 \mp j 3.4144) \times 10^{14}$ & $-(1.3005 \mp j 4.5288) \times 10^{12}$ \\
\hline
\end{tabular}

propagation, scattering and plasmon resonant effects of the graphene sheet.

\section{A. An Infinitely Large Graphene Sheet}

As the first example, an infinitely large free standing graphene sheet is benchmarked under the illumination of a normally incident Gaussian pulse. The temperature $T$ is set to be $T=300 \mathrm{~K}$, while $\mu_{c}$ and $\Gamma$ are two parameters to be determined.

Firstly, we set the chemical potential $\mu_{c}=0.3 \mathrm{eV}$ and the scattering rate $\Gamma=0.41 \mathrm{meV} / \hbar$. To facilitate the FRVF process, the surface conductivity from microwave to $\mathrm{THz}$ is fitted by rational functions using four poles. In Fig. 2, the comparison between the fitted value and the original data is shown from $500 \mathrm{MHz}$ to $10 \mathrm{THz}$. Excellent agreements are observed. The extracted poles and residues are listed in Table I. With these extracted values, the reflection $\Gamma_{R}$, transmission $\Gamma_{T}$ and absorption $\Gamma_{A}$ coefficients are calculated by the proposed DGTD-RBC algorithm, as shown in Fig. 3. For comparison, the analytical solutions calculated by method in [19] $\Gamma_{T}=1+\Gamma_{R}$ and $\Gamma_{A}=\sqrt{1-\left\|\Gamma_{T}\right\|^{2}-\left\|\Gamma_{R}\right\|^{2}}$ with $\Gamma_{R}=\frac{\eta_{2}-\eta_{1}-\sigma_{g} \eta_{1} \eta_{2}}{\eta_{2}+\eta_{1}+\sigma_{g} \eta_{1} \eta_{2}}$ are also presented, where $\eta_{1}$ and $\eta_{2}$ denote the characteristic impedances in region 1 and 2. It is noted that very good consistency is achieved. To investigate the $h$-convergence property of this proposed algorithm when using different mesh size, the error indicator defined by $\xi_{\text {err }}=10 \log \left(\left\|\Gamma_{\mathrm{DG}}-\Gamma_{\text {ref }}|| /|| \Gamma_{\mathrm{DG}}\right\|\right)$ is calculated and shown in Fig. 4. As it can be seen, the error exibts exponential decreasing.

Next, the transmission of a plane wave through the graphene sheet with $\mu_{c}=0.12 \mathrm{eV}$ and $\Gamma=2 \times 10^{12} \mathrm{~Hz}$ in the midinfrared region is studied. For FRVF, seven poles (see Table II) are employed with $500 \mathrm{MHz}$ sampling resolution from $500 \mathrm{GHz}$ to $100 \mathrm{THz}$. The fitted value and the original data are shown in Fig. 5. It is noted that $\sigma_{g}$ has a jump around $60 \mathrm{THz}$, thus more poles are needed. Below this frequency, the intraband dominates, otherwise interband overwhelms. The calculated amplitude of the transmission coefficient and the analytical result are presented in Fig. 6. Again, very good

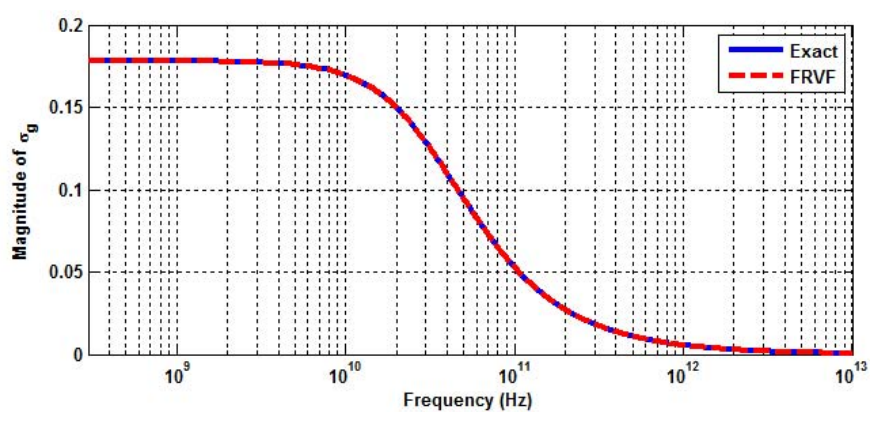

(a)

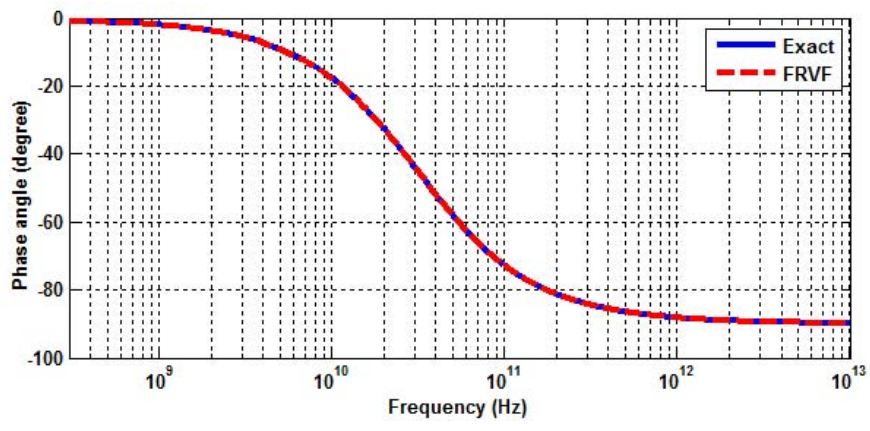

(b)

Fig. 2. The fitted magnitude (s) (a) and phase (b) of the surface conductivity $\sigma_{g}$ from $500 \mathrm{MHz}$ to $10 \mathrm{THz}$ with four poles.

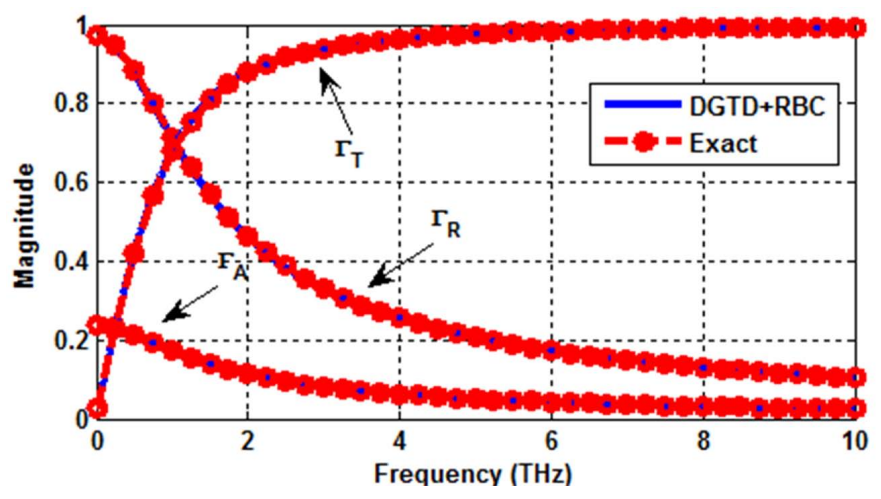

Fig. 3. The calculated magnitudes of coefficients $\Gamma_{T}, \Gamma_{R}$, and $\Gamma_{A}$ by the proposed algorithm as well as the theoretical data for $\mu_{c}=0.3 \mathrm{eV}$ case.

agreements between the numerical results and references are observed.

It is necessary to mention that the computational domain is truncated by the Silver-Müller absorbing boundary condition $(\mathrm{SM}-\mathrm{ABC})$ for the above simulations, which is rigorous since the wave is normally incident on the boundary.

\section{B. A Micro-Size Graphene Patch}

For finite-size graphene patch, the figure-of-merits of interest are the total-scattering cross-section (TSCS), the absorption cross-section (ACS), or the extinction cross-section (ECS). Another optional interesting parameter is the radar-crosssection (RCS) especially for people in the EM community. To truncate the computational domain, instead of using the $\mathrm{SM}-\mathrm{ABC}$, the time-domain boundary-integral (BI) equation is incorporated into the DGTD analysis [26], [27]. Based on the 


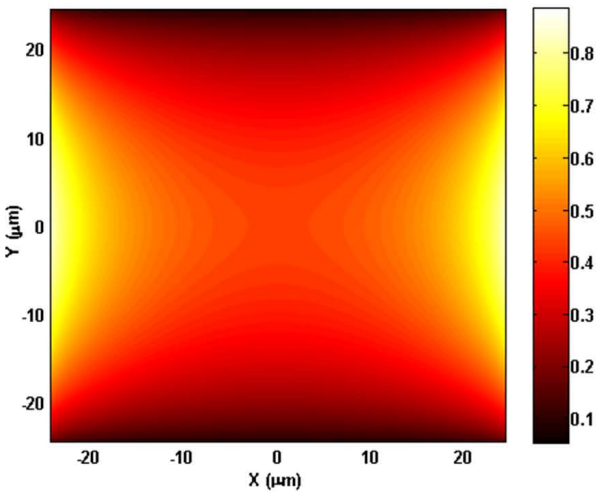

(a)

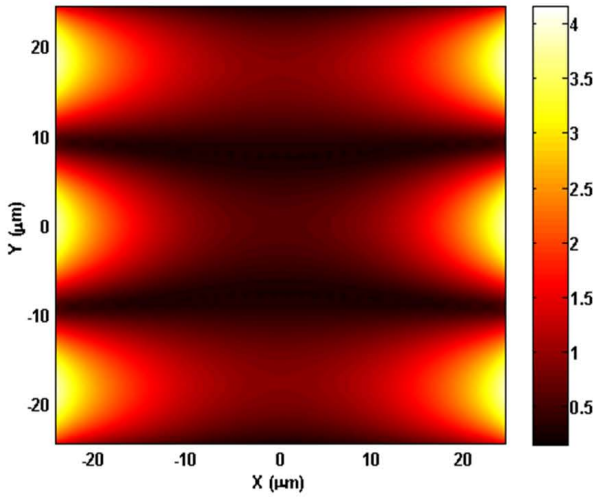

(b)

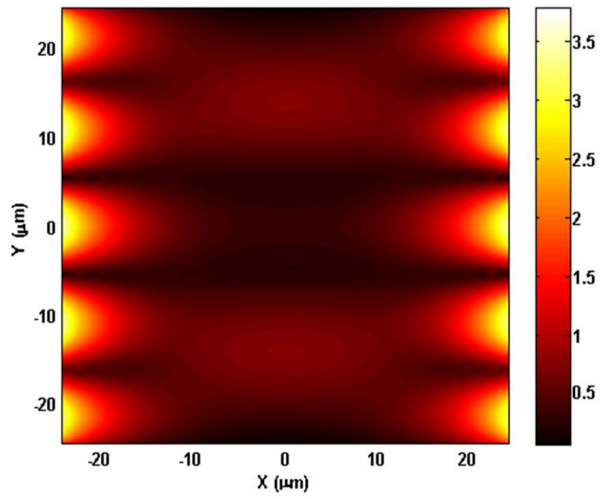

(c)

Fig. 9. The normalized near-field distribution of $E_{y}$ over the graphene sheet at $f_{1}=1.76 \mathrm{THz}(\mathrm{a}), f_{2}=4.98 \mathrm{THz}(\mathrm{b})$, and $f_{3}=6.97 \mathrm{THz}(\mathrm{c})$ for $\mu_{c}=1.5 \mathrm{eV}$ case.

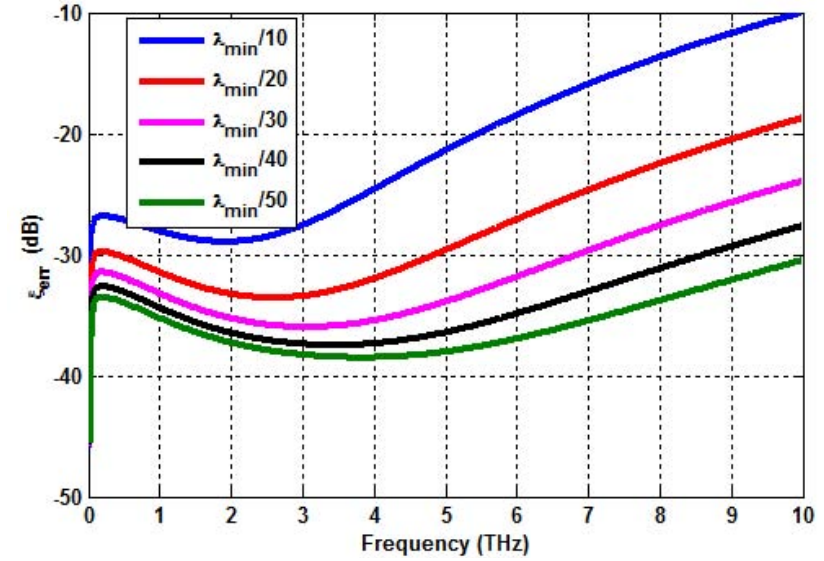

Fig. 4. The convergence property using different mesh cell sizes with $\lambda_{\min }$ denoting the free space wavelength at $10 \mathrm{THz}$.

TABLE III

The POLES $a_{m}$ AND RESIDUES $c_{m}$ FOR THE 5 B Y $10 \mu \mathrm{m}^{2}$ GRAPHENE SheET WITH $\mu_{c}=0 \mathrm{eV}$.

\begin{tabular}{ccc}
\hline \hline $\mathrm{m}$ & $a_{m}$ & $c_{m}$ \\
\hline 1 & $-4.287 \times 10^{13}$ & -3.268 \\
2 & $-1.000 \times 10^{13}$ & $4.219 \times 10^{9}$ \\
3 & $-1.021 \times 10^{10}$ & $-7.42 \times 10^{-4}$ \\
\hline
\end{tabular}

equivalent currents defined over a Huygens' surface, the field values required for evaluating the incoming numerical flux at the truncation boundary are calculated by the BI method. This method is mathematically exact and rigorous, i.e., the scattered waves coming from any direction can be absorbed perfectly.

To validate the proposed algorithm for $3 \mathrm{D}$ problem, a 5 by $10 \mu \mathrm{m}^{2}$ freestanding graphene patch in [25] is revisited. The parameters of the surface conductivity $\sigma_{g}$ are given by $T=300 \mathrm{~K}, \mu_{c}=0 \mathrm{eV}$, and $\Gamma=\frac{1}{2 \tau}$ with $\tau=10^{-13} \mathrm{~s}$. To facilitate the FRVF, the surface conductivity $\sigma_{g}$ is sampled from $500 \mathrm{MHz}$ to $5 \mathrm{THz}$ with the sampling resolution $f_{\text {step }}=500 \mathrm{MHz}$. Three poles and residues are adopted, which are listed in Table III. As for the excitation source, a Gaussian-modulated plane wave defined as $\mathbf{E}^{\text {inc }}(\mathbf{r}, t)=$ $\hat{\mathbf{y}} g\left(t-\hat{\mathbf{k}} \cdot \mathbf{r} / c_{0}\right)$ is used, where $\hat{\mathbf{k}}=\hat{\mathbf{z}}$ is the propagation

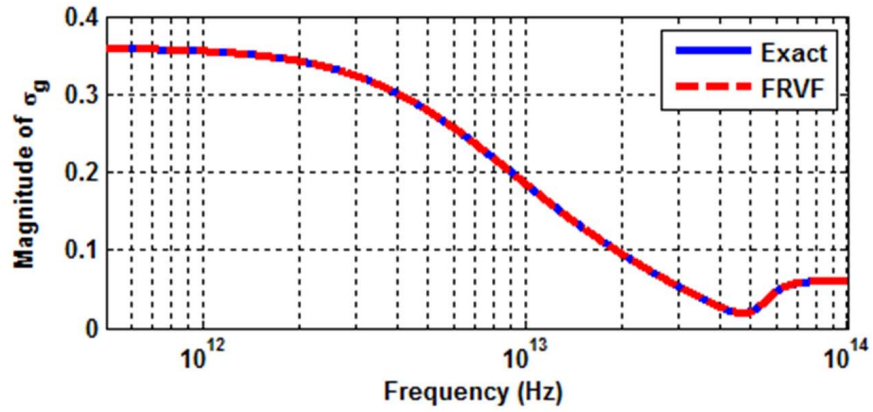

(a)

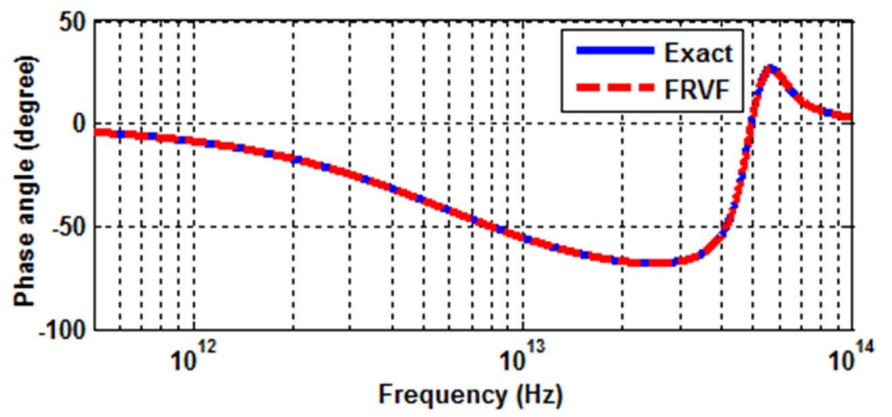

(b)

Fig. 5. The fitted magnitude (ms) (a) and phase (b) of the surface conductivity $\sigma_{g}$ from $500 \mathrm{GHz}$ to $100 \mathrm{THz}$ using seven poles.

direction, $g(t)=\exp \left(-\left[t-t_{0}\right]^{2} / \tau_{m}^{2}\right) \cos \left(2 \pi f_{m}\left[t-t_{0}\right]\right)$ is a Gaussian pulse with the modulation frequency $f_{m}=2.5$ $\mathrm{THz}$, duration $\tau_{m}=1.274 \times 10^{-13} \mathrm{~s}$, and delay $t_{0}=3 t_{m}$. For comparison, the normalized ECS from 0.1 to $4 \mathrm{THz}$ are calculated by the proposed DGTD-RBC algorithm, as shown in Fig. 7. The reference result in [25] obtained by integral equation method is also shown. The good agreements demonstrates the accuracy and applicability of the proposed algorithm for 3-D cases.

Next, a 50 by $50 \mu \mathrm{m}^{2}$ graphene patch with $T=300 \mathrm{~K}$ and $\Gamma=2.5 \mathrm{meV} / \hbar$ placed in the $x o y$ plane is characterized. Also, the same plane wave is employed as excitation but with $f_{m}=5 \mathrm{THz}$ and $\tau_{m}=6.37 \times 10^{-14} \mathrm{~s}$. 


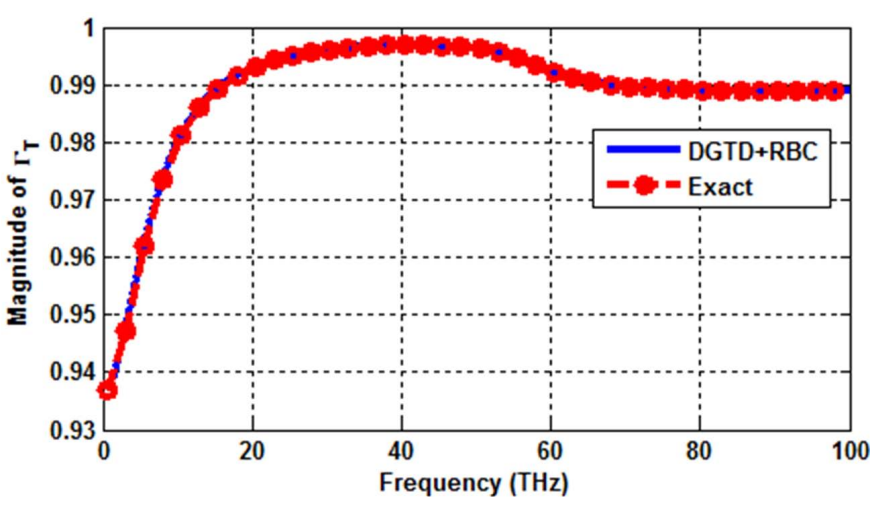

Fig. 6. The magnitude of calculated transmission coefficient $\Gamma_{T}$ by the proposed algorithm as well as theoretical data from $\mathrm{THz}$ to near-infrared region.

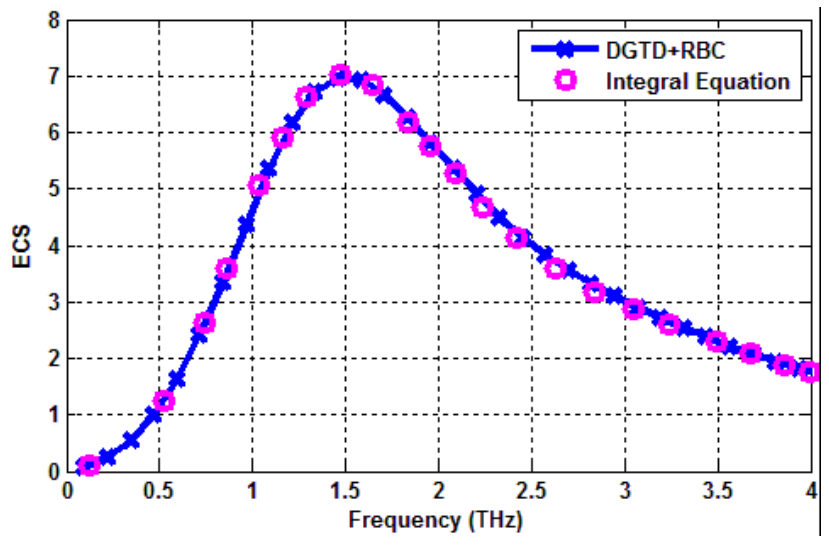

Fig. 7. The normalized ECS versus frequency for a freestanding graphene patch in [25] as its counterpart calculated by integral equation method.

Firstly, the TSCS and ACS of this graphene patch are evaluated for different chemical potentials $\mu_{c}$. There are totally 114, 404 tetrahedrons involved. The time step sizes for DGTD and $\mathrm{BI}$ are $\delta t_{D G}=1.073 \times 10^{-16} \mathrm{~s}$ and $\delta t_{B I}=2.45 \times 10^{-15}$ $\mathrm{s}$, respectively. For this example, 70,000 time steps are run. In Fig. 8, the normalized TSCS and ACS from 1 to $10 \mathrm{THz}$ are shown for $\mu_{c}=0.5,1.0,1.5 \mathrm{eV}$ (The poles and residues of the surface conductivity $\sigma_{g}$ are shown in the appendix). As expected, plasmon resonances arise at various frequencies. The increase of chemical potential results in the up-shift of resonant frequencies. The forward RCS from 1 to $10 \mathrm{THz}$ are also presented in Fig. 10. It is found that the peaks of RCS happen at the plasmon resonant frequencies, which is resulted from the near-field enhancement. In Fig. 9, the normalized near-field patterns of $E_{y}$ for $\mu_{c}=1.5 \mathrm{eV}$ case are plotted at three plasmon frequencies [see Fig. 8 (b)] $f_{1}=1.76 \mathrm{THz}$, $f_{2}=4.98 \mathrm{THz}$, and $f_{3}=6.97 \mathrm{THz}$. Noticeable near-field enhancement is observed at the resonant frequencies. In Fig. 11, the normalized far-field scattered patterns in E- and $\mathrm{H}$ planes at the fundamental plasmon frequency $f_{1}=1.76 \mathrm{THz}$ are also presented. It is interestingly found that the far-field patterns resemble those of conventional short dipoles [28], which consists with the assertion in [28]. The non-symmetrical pattern in $\mathrm{H}$-plane is attributed to the boundary condition in (6).

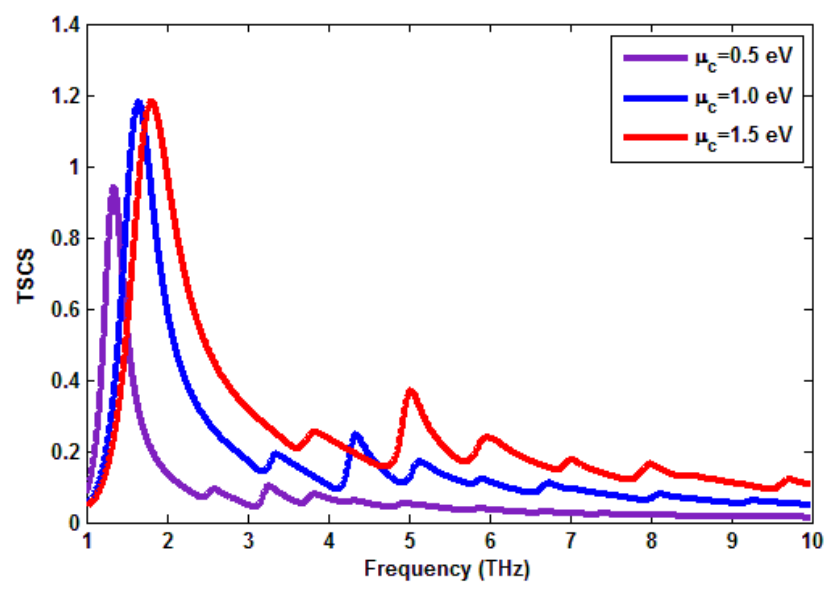

(a)

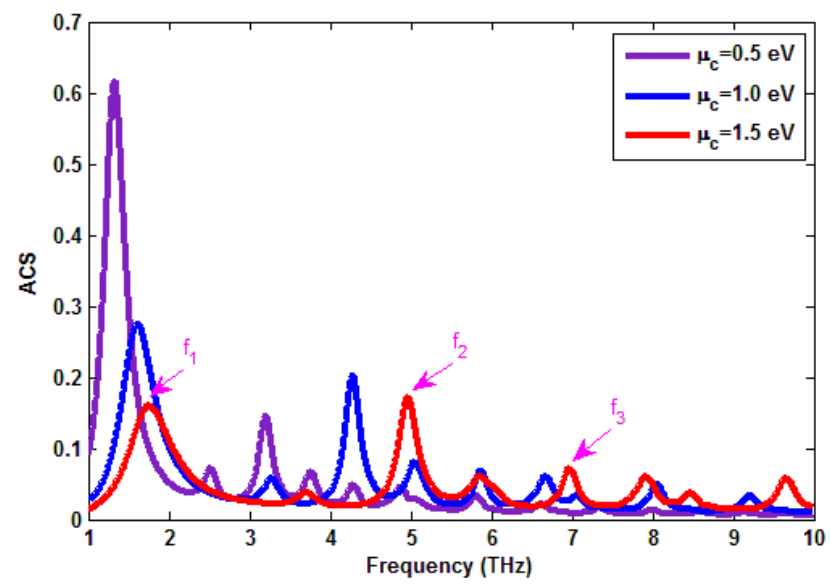

(b)

Fig. 8. The TSCS (a) and ACS (b) of the 50 by $50 \mu \mathrm{m}^{2}$ freestanding graphene patch corresponding to different chemical potentials $\mu_{c}=$ $0.5,1.0,1.5 \mathrm{eV}$.

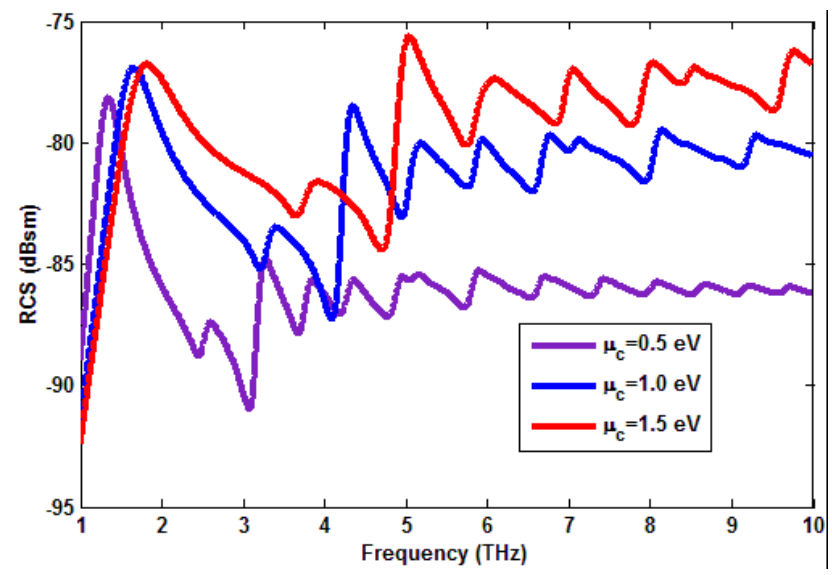

Fig. 10. The calculated forward RCS of the 50 by $50 \mu \mathrm{m}^{2}$ freestanding graphene patch corresponding to chemical potentials $\mu_{c}=0.5,1.0,1.5 \mathrm{eV}$.

To have a basic insight into the effect of substrate on the plasmon resonance, we suppose that the above graphene patch is supported by a $1 \mu$ m-thick dielectric substrate. Three dielectric mediums that are familiar to experimental researchers are considered, including silicon-dioxide $\left(\mathrm{SiO}_{2}\right)$ with $\epsilon_{r}=4.0$, silicon-nitrate $\left(\mathrm{Si}_{3} \mathrm{~N}_{4}\right)$ with $\epsilon_{r}=7.0$, and 


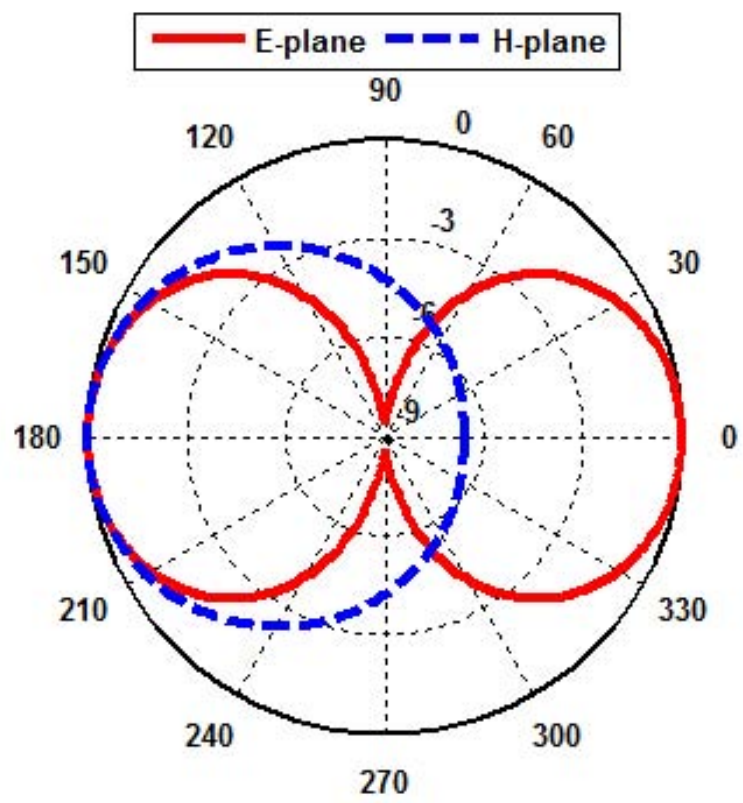

Fig. 11. The normalized far-field pattern in E- and H-plane at $f_{1}=1.76$ THz for $\mu_{c}=1.5 \mathrm{eV}$ case.

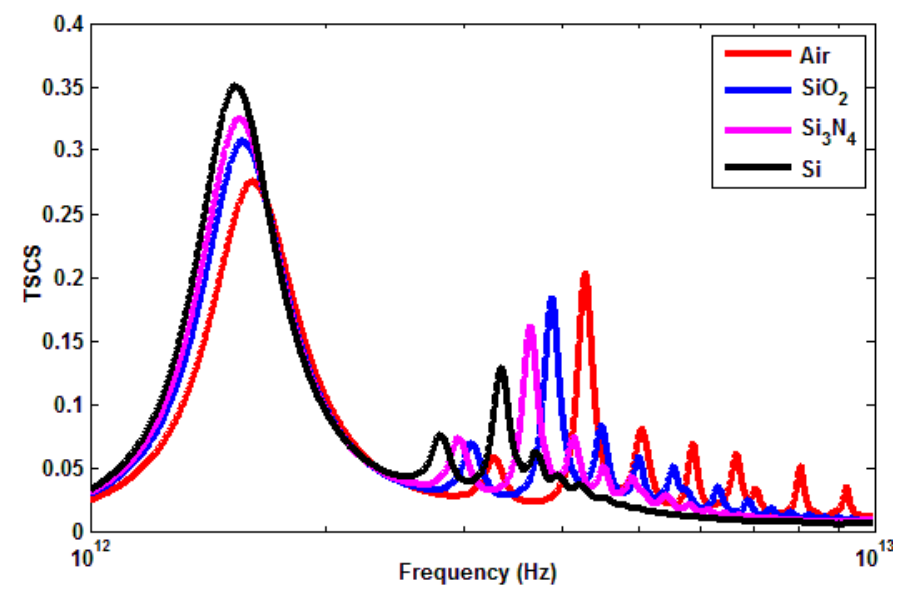

Fig. 12. The normalized ACS of a 50 by $50 \mu \mathrm{m}^{2}$ graphene patch supported by different dielectric substrates for $\mu_{c}=1 \mathrm{eV}$.

silicon (Si) with $\epsilon_{r}=11.9$. Fig. 12 shows the obtained ACS by the proposed algorithm for $\mu_{c}=1.0 \mathrm{eV}$. It is interestingly noted that the plasmon resonance shifts to low frequencies with the increasing of permittivity and more energy is collected at the fundament plasmon mode while the high order modes are suppressed. The reasons behind these phenomenons are attributed to higher effective permittivity resulting lower resonance frequencies and stronger confinements of surface plasmon waves. In Fig. 13, the normalized magnetic near-fields $H_{x}$ over the graphene at $f_{1}=1.57 \mathrm{THz}$ and $f_{2}=3.89 \mathrm{THz}$ for silicon-dioxide case are shown. Obvious discontinuities are noted over the two sides of the graphene.

\section{Graphene-Strip Gratings}

For the third example, five 5 by $50 \mu \mathrm{m}^{2}$ graphene strips are parallel placed along the $y$-axis with period $p$. Here, the scattering rate and chemical potential are set to be $\mu_{c}=1.0$

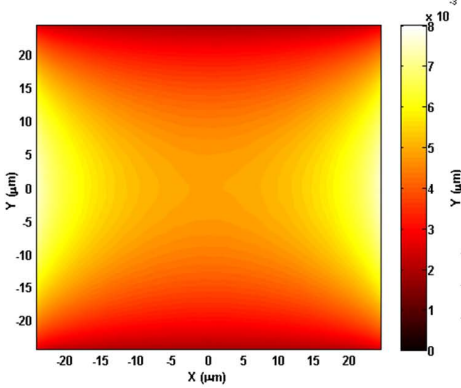

(a)

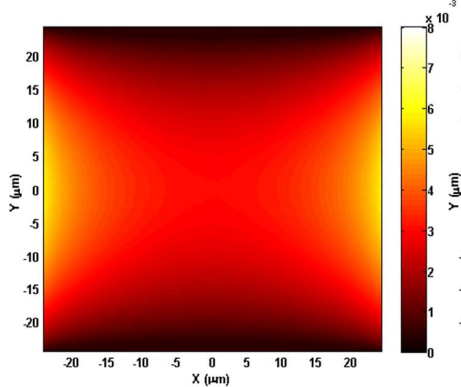

(c)

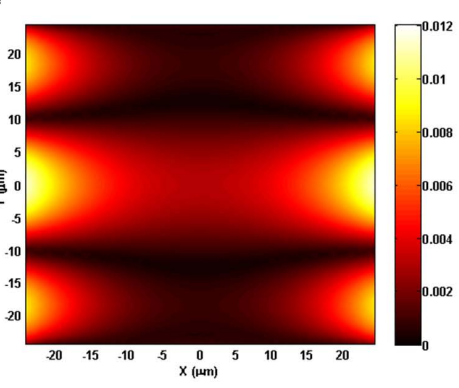

(b)

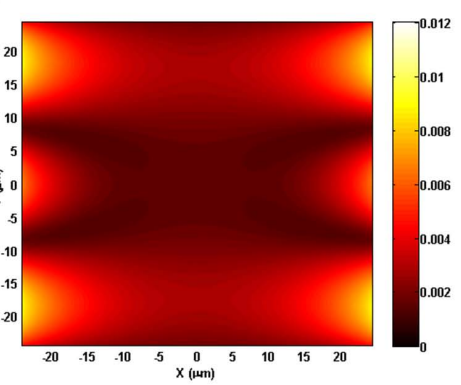

(d)
Fig. 13. The normalized magnetic field $H_{x}$ over the two sides of the graphene sheet at $f_{1}=1.57 \mathrm{THz}$ (a)-(c) and $f_{2}=3.89 \mathrm{THz}$ (b)-(d) for the silicondioxide case.

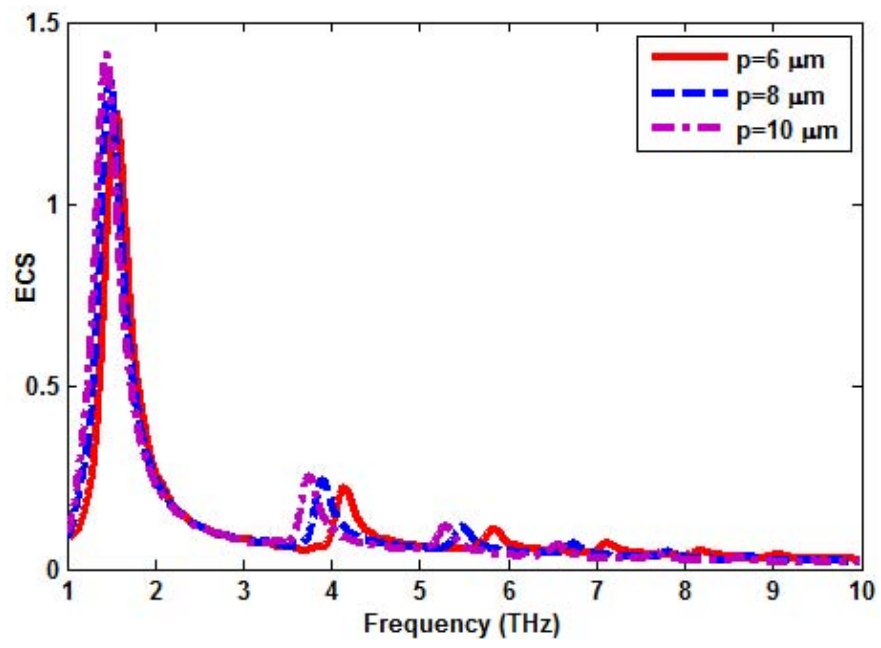

Fig. 14. The normalized ECS of graphene gratings with period $p=6,8,10$ $\mu \mathrm{m}$.

$\mathrm{eV}$ and $\Gamma=2.5 \mathrm{meV} / \hbar$, respectively. Also, the previous Gaussian plane wave is employed as the excitation but with $x$-polarization.

In this benchmark, the impacts of different grating period $p$ on the plasmon resonance are investigated. In Table IV, the time step size $\delta t_{D G}, \delta t_{B I}$, and the number of mesh elements $N$ for $p=6,8,10 \mu \mathrm{m}$ are listed. The normalized ECS for different period $p$ are shown in Fig. 14. It is noted that the plasmon frequencies shift downward as the increasing of the period and simultaneously smaller number of plasmon modes are excited. In Fig. 15, the total electric field over the graphene plane for the first three resonant frequencies with $p=10 \mu \mathrm{m}$ are presented. The propagation of surface plasmon wave is 

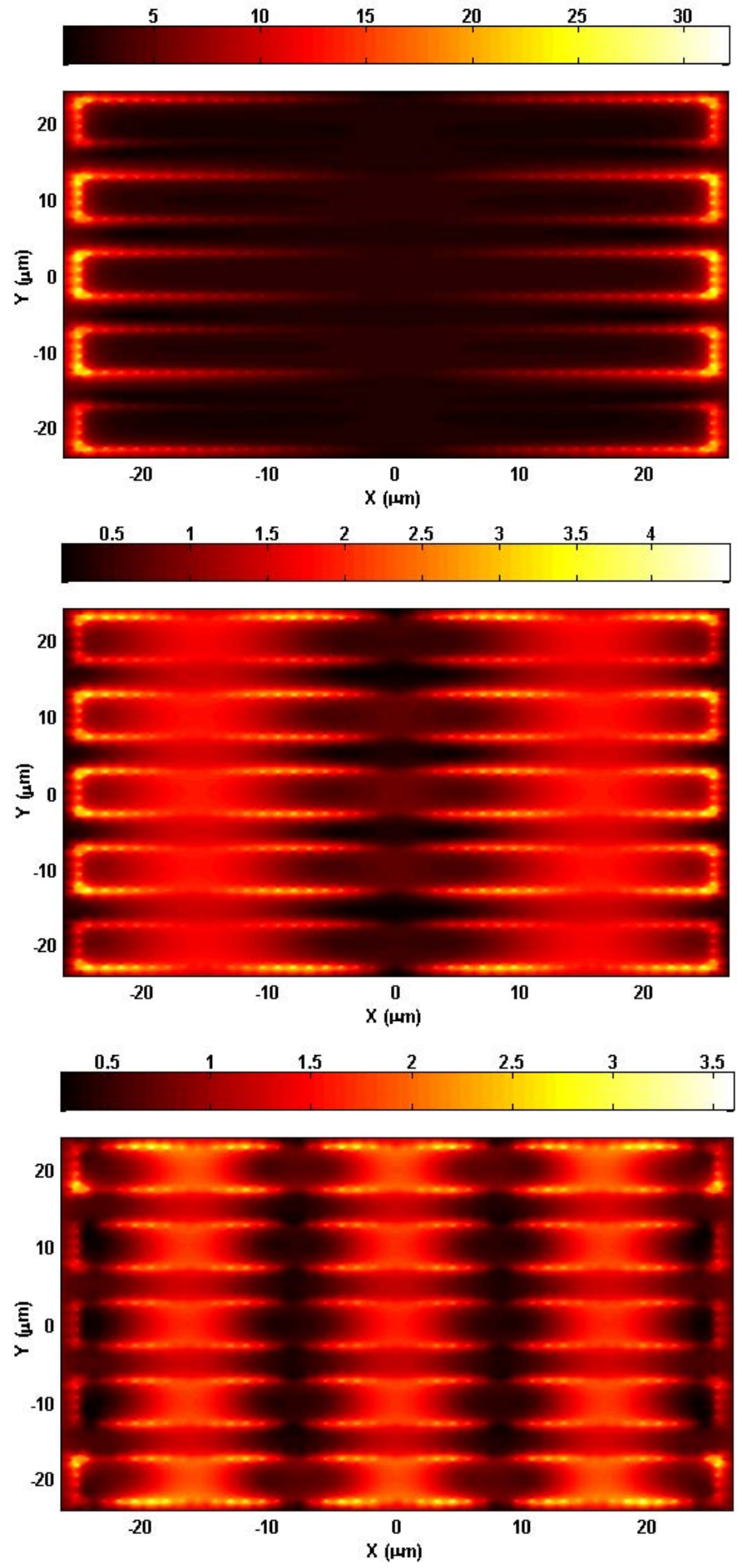

Fig. 15. The normalized electric field over the graphene plane at resonant frequencies $f_{1}=1.445 \mathrm{THz}, f_{2}=3.735 \mathrm{THz}$, and $f_{3}=5.291 \mathrm{THz}$ for period $p=10 \mu \mathrm{m}$ case.

clearly observed.

\section{CONCLUSION}

In this paper, a DGTD based algorithm is developed to model the graphene described by a surface conductivity $\sigma_{g}$. Instead of volumetrically meshing the atomically-thick graphene, a R$\mathrm{BC}$ is applied by considering the graphene as an infinitely thin
TABLE IV

THE TIME STEP SIZE $\delta t_{D G}$ FOR DG AND $\delta t_{B I}$ FOR BI AND THE NUMBER OF MESH ELEMENTS $N$.

\begin{tabular}{clcc}
\hline \hline$p(\mu \mathrm{m})$ & $\delta t_{D G}(s)$ & $\delta t_{B I}(s)$ & $N$ \\
\hline 6 & $6.25 \times 10^{-17}$ & $1.45 \times 10^{-17}$ & 85,128 \\
8 & $7.92 \times 10^{-17}$ & $1.52 \times 10^{-15}$ & 94,501 \\
10 & $7.01 \times 10^{-17}$ & $1.55 \times 10^{-15}$ & 99,988 \\
\hline
\end{tabular}

TABLE V

The Poles $a_{m}$ AND Residues $c_{m}$ FOR THE GRAPHene SheEt With $\mu_{c}=0.5 \mathrm{eV}$.

\begin{tabular}{ccc}
\hline \hline $\mathrm{m}$ & $a_{m}$ & $c_{m}$ \\
\hline 1 & $-1.351 \times 10^{10}$ & 0.0655 \\
2 & $-1.209 \times 10^{12}$ & $1.177 \times 10^{11}$ \\
3 & $-(0.0012-j 2.635) \times 10^{15}$ & $8.852 \times 10^{10}+j 5.297 \times 10^{5}$ \\
4 & $-(0.0012+j 2.635) \times 10^{15}$ & $8.852 \times 10^{10}-j 5.297 \times 10^{5}$ \\
\hline
\end{tabular}

TABLE VI

THE POLES $a_{m}$ AND RESIDUES $c_{m}$ FOR THE GRAPHENE SHEET WITH $\mu_{c}=1.0 \mathrm{eV}$.

\begin{tabular}{ccc}
\hline \hline $\mathrm{m}$ & $a_{m}$ & $c_{m}$ \\
\hline 1 & $-1.395 \times 10^{9}$ & 0.9325 \\
2 & $-1.209 \times 10^{12}$ & $1.177 \times 10^{11}$ \\
3 & $-(0.0013-j 5.289) \times 10^{15}$ & $1.783 \times 10^{11}+j 1.588 \times 10^{6}$ \\
4 & $-(0.0013+j 5.289) \times 10^{15}$ & $1.783 \times 10^{11}-j 1.588 \times 10^{6}$ \\
\hline
\end{tabular}

TABLE VII

THE POLES $a_{m}$ AND RESIDUES $c_{m}$ FOR THE GRAPHENE SHEET With $\mu_{c}=1.5 \mathrm{eV}$.

\begin{tabular}{ccc}
\hline \hline $\mathrm{m}$ & $a_{m}$ & $c_{m}$ \\
\hline 1 & $-1.561 \times 10^{8}$ & -0.1366 \\
2 & $-1.209 \times 10^{12}$ & $1.766 \times 10^{11}$ \\
3 & $-(0.00094-j 7.808) \times 10^{15}$ & $2.591 \times 10^{11}-j 8.879 \times 10^{6}$ \\
4 & $-(0.00094+j 7.808) \times 10^{15}$ & $2.591 \times 10^{11}+j 8.879 \times 10^{6}$ \\
\hline
\end{tabular}

conductive sheet with the surface conductivity $\sigma_{g}$ described by the Kubo-formula. To incorporate this boundary condition into DGTD analysis, the formulation of numerical upwind flux is rederived based on the Rankine-Hugoniot jump relations. By approximating the surface conductivity with rational functions using the FRVF technique, the corresponding time-domain matrix equations can be obtained in an integral form over time $t$ via the inverse Laplace transform. The integral operators are discretized by FIT. The validation and applicability of the proposed algorithm are demonstrated by a set of numerical examples.

\section{APPENDIX A}

The Residues and Poles of the Rational Functions CORRESPONDING TO DIFFERENT $\mu_{c}$

This part shows the poles and residues of the rational functions used to approximate the surface conductivity $\sigma_{g}$ of the graphene patch corresponding to different chemical potentials $\mu_{c}$. To facilitate the FRVF technique, the sampling is conducted from $500 \mathrm{MHz}$ to $10 \mathrm{THz}$ with sampling resolution $f_{\text {step }}=500 \mathrm{MHz}$. The number of poles and residues are set to four, as shown in the following tables. 


\section{REFERENCES}

[1] P. Sharma, J. P. Carrier, C. Moldovan, and A. M. Ionescu, "Electromagnetic performance of RF NEMS graphene capacitive switches," IEEE Trans. Nanotechn., vol. 13, no. 1, pp. 70-79, Jan. 2014.

[2] A. Vakil and Engheta, "Transformation optics using graphene," Science, vol. 332, pp. 1291-1295, Jun. 2011.

[3] G. D. Bouzianas, N. V. Kantartzis, and T. D. Tsiboukis, "Plasmon mode excitation on graphene layers via obliquely-incident focused wideband pulses in rigorous time-domain algorithms," IEEE Trans. Magn., vol. 49, no. 5, pp. 1773-1776, May 2013.

[4] G. W. Hanson, "Dyadic Green's functions for an anisotropic, non-Local model of biased graphene," IEEE Trans. Antennas Propag., vol. 56, no. 3, pp. 747-757, Mar. 2008.

[5] G. Lovat, "Equivalent circuit for electromagnetic interaction and transmission through graphene sheets," IEEE Trans. Electromag. Compat., vol. 54, no. 1, pp. 101-109, Feb. 2012.

[6] D. C-Serrano, J. S. G-Diaz, J. P-Carrier, and A. A-Melcon, "Spatially dispersive graphene single and parallel plate waveguides: analysis and circuit model," IEEE Trans. Microw. Theory Techn., vol. 61. no. 12, pp. 4333-4344, Dec. 2013.

[7] O. V. Shapoval, J. S. G-Diaz, J. P-Carrier, J. R. Mosig, and A. I. Nosich, "Integral equation analysis of plane wave scattering by coplanar graphenestrip gratings in the THz range," IEEE Trans. Terahertz Sci. Techn., vol. 6, no. 3, pp. 666-674, Sept. 2013.

[8] V. Nayyeri, M. Soleimani, and M. Ramahi, "Wideband modeling of graphene using the finite-difference time-domain metod," IEEE Trans. Antennas Propag., vol. 6, no. 12, pp. 6107-6114, Dec. 2013.

[9] V. Nayyeri, M. Soleimani, and M. Ramahi, "Modeling graphene in the finite-difference time-domain method using a surface boundary condition," IEEE Trans. Antennas Propag., vol. 6, no. 8, pp. 4176-4182, Aug. 2013.

[10] H. Lin, M. F. Pantoja, L. D. Angulo, J. Alvarez, R. G. Martin, and S. G. Garcia, "FDTD modeling of graphene devices using complex conjugate dispersion material model," IEEE Microw. Wirelss Compon. Lett., vol. 22 , no. 12 , Dec. 2012.

[11] I. Ahmed, E. H. Khoo, and E. Li, "Efficient modeling and simulation of graphene devices with the LOD-FDTD method," IEEE Microw. Wireless Compon. Lett., vol. 23, no. 6, pp. 306-308, Jun. 2013.

[12] K. Sankaran, "Accurate domian truncation techniques for time-domain conformal methods," Ph.D. dissertation, Dept. Inform. Technol. Elect. Eng., ETH Zurich, Zurich, Switzerland, 2007.

[13] J. M. Jin, The Finite Element Method in Electromagnetics, 2nd ed. New York, NY, USA: Wiley, 2003.

[14] B. Gustavsen and A. Semlyen, "Rational approximation of frequency domian responses by vector fitting," IEEE Trans. Power Del., vol. 14, no. 3, pp. 1052-1061, Jul. 1999.

[15] B. Gustavsen, "Improving the pole relocating properties of vector fitting," IEEE Trans. Power Del., vol. 21, no. 3, pp. 1587-1592, Jul. 2006.

[16] P. Li and L. J. Jiang, "Integration of arbitrary lumped multiport circuit networks into discontinuous Galerkin time-domain analysis," IEEE Trans. Microw. Theory and Techn., vol. 61, no. 7, pp. 2525-2534, Jul. 2013.

[17] S. D. Gedney, J. C. Young, T. C. Kramer, and J. A. Roden, "A discontinuous Galerkin finite element time-domain method modeling of dispersive media," IEEE Trans. Antennas Propag., vol. 60, no. 4, pp. 1969-1977, Apr. 2012.

[18] P. Li, L. J. Jiang, and H. Bağcı, "An IBC enhanced DGTD scheme for transient analysis of EM interactions with graphene," in IEEE Int. Symp. Antennas Propag., pp. 1393-1394, Jul. 6-11, 2014.

[19] G. Hanson, "Dyadic Green's functions and guided waves for a surface conductivity model of graphene," J. Appl. Phys., vol. 103, no. 064302, Mar. 2008.

[20] P. Li and L. J. Jiang, "A hybrid electromagnetics-circuit simulation method exploiting discontinuous Galerkin finite element time domain method," IEEE Microw. Wireless Compon. Lett., vol. 23, no. 3, pp. 113115, Mar. 2013.

[21] P. Li and L. J. Jiang, "Simulation of electromagnetic waves in the magnetized cold plasma by a DGFETD method," IEEE Antenna Wireless Propag. Lett., vol. 12, pp. 1244-1247, Dec. 2013.

[22] S. D. Gedney, C. Luo, J. A. Roden, R. D. Crawford, B. Guernsey, J. A. Miller, T. Kramer, and E. W. Lucas, "The discontinuous Galerkin finite element time-domain method solution of Maxwell's equations," J. Appl. Comput. Electromagn. Society, vol. 24, no. 2, pp. 129-142, Apr. 2009.

[23] S. Dosopoulos, B. Zhao, and J. F. Lee, "Non-conformal and parallel discontinuous Galerkin time domain method for Maxwell's equations: EM analysis of IC packages," J. Comput. Phys., vol. 238, no. 12, pp. 48-70, Dec. 2012.
[24] J. S. Hesthaven and T. Warburton, Nodal Discontinuous Galerkin Methods. Berlin: Springer, 2008

[25] I. Llatser, C. Kremers, A. C.-Aparicio, J. M. Jornet, E. Alarcon, and D. N. Chigrin,"Graphene-based nano-patch antenna for terahertz radiation," Photonics Nanostructures: Fundamentals and Applications, vol. 10, pp. 353-358, May 2012.

[26] P. Li, Y. Shi, L. J. Jiang, and H. Bağcı, "A discontinuous Galerkin time domain-boundary integral method for analyzing transient electromagnetic scattering," in IEEE Int. Symp. Antennas Propag., pp. 1893-1894, Jul. 611,2014

[27] P. Li, Y. Shi, L. J. Jiang, and H. Bağcı, "A hybrid time-domain discontinuous galerkin-boundary integral method for electromagnetic scattering analysis," IEEE Trans. Antennas Propag., vol. 62, no. 5, pp. 2841-2846, May 2014.

[28] M. Tamagnone, J. S. Gomez-Diaz, J. R. Mosig, and J. P. Carrier, "Analysis and design of terahertz antennas based on plasmonic resonant graphene sheets," Appl. Phys. Lett., vol. 101, no. 214102, pp. 1-4, 2012.

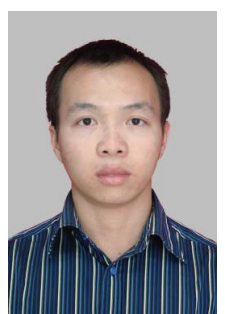

Ping Li (S'12) has been working toward the Ph.D. degree with the center of Electromagnetics and Optics at the University of Hong Kong since 2010. $\mathrm{He}$ is the recipient of the Postgraduate Engineering Fellowship from 2010. He is listed in Marquis Who's Who in the World, $32^{\text {nd }}$ Edition, 2015.

He has authored over 10 journal papers on IEEE Trans. Microwave Theory and Techniques, IEEE Trans. Antennas and Propagation, IEEE Trans. Electromagnetic and Compatibility, IEEE Trans. Components, Packaging, and Manufacturing Technologies, etc. His paper was selected as the Finalist paper in 29-th International Review of Progress in Applied Computational Electromagnetics and 2014 International Symposium on Electromagnetic Compatibility, and he won the best student paper award in 12-th International Workshop on Finite Elements for Microwave Engineering. He served as the reviewer of IEEE Trans. Antennas and Propagation, IEEE Antennas and Wireless Propagation Letters, Proceedings of IEEE, and ACES Journal.

His research interests include the near-field to far-field transformation techniques, phaseless equivalent source reconstruction methods, discontinuous Galerkin time-domain method, and uncertainty quantification for large scale electromagnetic systems. 


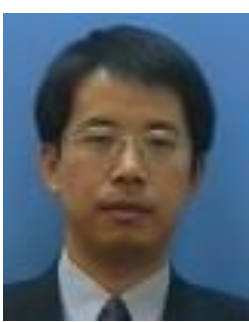

Li Jun Jiang (S'01-M'04-S'13) received the B.S. degree in electrical engineering from the Beijing University of Aeronautics and Astronautics in 1993, the M.S. degree from the Tsinghua University in 1996, and P.h.D from the University of Illinois at Urbana-Champaign in 2004. From 1996 to 1999 , he was an application engineer with the HewlettPackard Company. Since 2004, he has been the postdoctoral researcher, the research staff member, and the senior engineer at IBM T.J. Watson Research Center. Since the end of 2009, he is an Associate Professor with the Department of Electrical and Electronic Engineering at the University of Hong Kong.

He received the IEEE MTT Graduate Fellowship Award in 2003 and the Y.T. Lo Outstanding Research Award in 2004. He is an IEEE Senior Member, the Associate Editor of IEEE Transactions on Antennas and Propagation, the Associate Editor of Progress in Electromagnetics Research, the Associate Guest Editor of the Proceedings of IEEE Special Issue in 2011 2012, an IEEE AP-S Member, an IEEE MTT-S member, an IEEE EMC-S member, an ACES member, and a member of Chinese Computational Electromagnetics Society. He was the Semiconductor Research Cooperation (SRC) Industrial Liaison for several academic projects. He served as the Scientific Consultant to Hong Kong ASTRI (Hong Kong Applied Science and Technology Research Institute Company Limited) in 2010-2011, the Panelist of the Expert Review Panel (ERP) of Hong Kong R\&D Centre for Logistics and Supply Chain Management Enabling Technologies since Jan. 1st, 2013. He is also the senior visiting professor at Tsinghua University since Jun. 2013.

He was the TPC Chair of the 7th International Conference on Nanophotonics (ICNP)/the 3rd Conference on Advances in Optoelectronics and Micro/Nano Optics (AOM), the TPC Co-chair of the 12th International Workshop on Finite Elements for Microwave Engineering, the Co-chair of 2013 International Workshop on Pulsed Electromagnetic Field at Delft, the Netherlands, the General Chair of 2014 IEEE 14th HK AP/MTT Postgraduate Conference. He was the elected TPC member of IEEE EPEP since 2014 the TPC member of IEEE EDAPS since 2010, the TPC member of 2013 IEEE ICMTCE, the scientific committee member of 2010 IEEE SMEE, the special session organizers of IEEE EDAPS, IEEE EMC, ACES, APRASC, PIERS, co-organizer of HKU Computational Science and Engineering Workshops in 2010-2012, the TC-9 and TC-10 member of IEEE EMC-S since 2011, and session chairs of many international conferences. He also serves as the reviewer of IEEE Transactions on several topics, and other primary electromagnetics and microwave related journals.

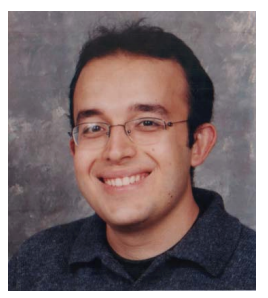

Hakan Bağcii (S'98-M'07) received the B.S. degree in Electrical and Electronics Engineering from the Bilkent University, Ankara, Turkey, in June 2001 and the M.S. and Ph.D. degrees in Electrical and Computer Engineering from the University of Illinois at Urbana-Champaign (UIUC), Urbana, in August 2003 and January 2007, respectively. From June 1999 to July 2001, he worked as an Undergraduate Researcher at the Computational Electromagnetics Group, Bilkent University. From August 2001 to December 2006, he was a Research Assistant at the Center for Computational Electromagnetics and Electromagnetics Laboratory, UIUC. From January 2007 to August 2009, he worked as a Research Fellow at the Radiation Laboratory, University of Michigan. In August 2009, he joined the Division of Physical Sciences and Engineering at the King Abdullah University of Science and Technology (KAUST) as Assistant Professor of Electrical Engineering. His research interests include various aspects of computational electromagnetics with emphasis on time-domain integral equations and their fast marching-on-in-time-based solutions, well-conditioned integralequation formulations, and development of fast hybrid methods for analyzing statistical EMC/EMI phenomena on complex and fully loaded platforms.

Dr. Bağcı was the recipient of the 2008 International Union of Radio Scientists (URSI) Young Scientist Award and the 2004-2005 Interdisciplinary Graduate Fellowship from the Computational Science and Engineering Department, UIUC. His paper titled "Fast and rigorous analysis of EMC/EMI phenomena on electrically large and complex structures loaded with coaxial cables" was one of the three finalists (with honorable mention) for the 2008 Richard B. Schulz Best Transactions Paper Award given by the IEEE Electromagnetic Compatibility Society. He authored and co-authored eight finalist papers in the student paper competitions at the 2005, 2008, and 2010 IEEE Antennas and Propagation Society International Symposiums and 2013 and 2014 Applied Computational Electromagnetics Society Conference. 